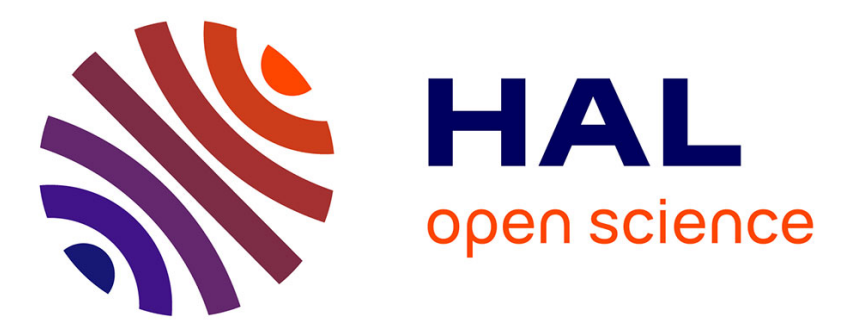

\title{
La demande de raccordement des ménages auprès des petits opérateurs privés d'eau potable
}

\author{
Anne Briand, Amandine Loyal Laré-Dondarini
}

\section{To cite this version:}

Anne Briand, Amandine Loyal Laré-Dondarini. La demande de raccordement des ménages auprès des petits opérateurs privés d'eau potable: Le cas des quartiers périphériques de Maputo. Revue Economique, 2013. hal-02430272

\section{HAL Id: hal-02430272 \\ https://hal.science/hal-02430272}

Submitted on 7 Jan 2020

HAL is a multi-disciplinary open access archive for the deposit and dissemination of scientific research documents, whether they are published or not. The documents may come from teaching and research institutions in France or abroad, or from public or private research centers.
L'archive ouverte pluridisciplinaire HAL, est destinée au dépôt et à la diffusion de documents scientifiques de niveau recherche, publiés ou non, émanant des établissements d'enseignement et de recherche français ou étrangers, des laboratoires publics ou privés. 


\section{LA DEMANDE DE RACCORDEMENT DES MÉNAGES AUPRÈS DES PETITS OPÉRATEURS PRIVÉS D'EAU POTABLE}

Le cas des quartiers périphériques de Maputo

\section{Anne Briand et Amandine Loyal}

Presses de Sciences Po | « Revue économique »

2013/4 Vol. 64 | pages 685 à 719

ISSN 0035-2764

ISBN 9782724633016

Article disponible en ligne à l'adresse :

https://www.cairn.info/revue-economique-2013-4-page-685.htm

Distribution électronique Cairn.info pour Presses de Sciences Po.

(C) Presses de Sciences Po. Tous droits réservés pour tous pays.

La reproduction ou représentation de cet article, notamment par photocopie, n'est autorisée que dans les limites des conditions générales d'utilisation du site ou, le cas échéant, des conditions générales de la licence souscrite par votre établissement. Toute autre reproduction ou représentation, en tout ou partie, sous quelque forme et de quelque manière que ce soit, est interdite sauf accord préalable et écrit de l'éditeur, en dehors des cas prévus par la législation en vigueur en France. Il est précisé que son stockage dans une base de données est également interdit. 


\title{
La demande de raccordement des ménages auprès des petits opérateurs privés d'eau potable
}

\author{
Le cas des quartiers périphériques de Maputo
}

\author{
Anne Briand* \\ Amandine Loyal Laré*
}

\begin{abstract}
L'article vise à analyser le choix de raccordement des ménages aux réseaux spontanés d'eau potable développés par des petits opérateurs privés (POPS) dans les quartiers périphériques de Maputo (Mozambique) grâce aux données d'une enquête réalisée auprès de 880 ménages. L'estimation de modèles Probit permet de dégager des résultats nouveaux sur l'accès à l'eau des ménages en Afrique. En particulier, nous montrons que les quartiers périphériques de la capitale sont hétérogènes et que les réseaux développés par les POPS ne sont toujours pas accessibles aux ménages les plus pauvres (vivant avec moins d'un dollar par jour et par personne).
\end{abstract}

\section{HOUSEHOLDS' DEMAND OF TAP CONNECTION FROM SMALL- SCALE PRIVATE OPERATORS: A STUDY APPLIED TO MAPUTO'S PERIPHERAL DISTRICTS}

The purpose of this article is to explain the choice of private water connection by urban households in peripheral districts using microdata from Maputo (Mozambique). Using data on 880 households, we estimate probit models. Results contribute to the literature on households' water supply in Africa. In particular, we show that the peripheral districts are heterogeneous and the private connections developed by the small-scale private operators are not yet used by the poorest households (income below one dollar per day per person).

Classification JEL : Q25, R21, O55

\footnotetext{
*CREAM (Centre de Recherche en Économie Appliquée à la Mondialisation), UFR DroitÉconomie-Gestion.Correspondance : 3 avenue Pasteur, 76000 Rouen.Courriels : anne.briand@ univ-rouen.fr; loyallare@gmail.com.

Les auteurs remercient l'AFD pour le financement de l'enquête et, en particulier, Janique Étienne pour tous ses conseils.
} 


\section{INTRODUCTION}

Les petits opérateurs privés (POPs) d'eau potable représentent, depuis les années 1970, un objet d'étude important, en particulier dans les centres périurbains des grandes agglomérations des pays en développement (Hart [1970]). Face à un secteur public défaillant, des acteurs indépendants issus de la société civile développent différents niveaux de services d'eau potable auprès des ménages (Collignon [1998]). Des travaux montrent que la participation des opérateurs privés permet d'augmenter substantiellement les taux d'accès à l'eau potable aussi bien en Amérique latine (Solo [2003] ; Clarke, Kosec et Wallsten [2004]), en Asie (Conan [2003]) qu'en Afrique (Collignon et Vezina [2000]). Contrairement à l'étude menée par Budds et McGranahan [2003], les auteurs défendent généralement l'idée selon laquelle les POPs représentent une opportunité pour l'atteinte des objectifs du millénaire (McGranahan, Njiru, Albu, Smith et Mitlin [2006]). Plus particulièrement, les opérateurs privés auraient une plus grande capacité que le secteur public à cibler les populations à faible revenu, ce qui entraîne parfois un certain nombre d'effets contre-redistributifs (Conan et Paniagua, [2003] ; McGranahan et Owen [2006] ; Franceys [1997] et Franceys et Weitz [2003]). Cette intuition, qui fait débat depuis une dizaine d'années, reste une question de recherche centrale dans la mesure où l'enjeu majeur des agglomérations du Sud, dont la population ne cesse de croître (compte tenu de l'exode rural et de la croissance démographique), demeure l'accès aux services de base tels que l'approvisionnement en eau potable. Ce problème d'accès des populations, notamment des plus pauvres, représente le cœur des discussions abondamment abordées dans la littérature en lien avec les questions des bienfaits ou non de la privatisation du secteur, de la régulation des partenariats public-privé et des modes de gouvernance à instaurer (Whittington [2003]). Si la littérature a également étudié les déterminants du choix des ménages entre les différents systèmes d'approvisionnement en eau, l'existence d'un service, tel que celui fourni par les petits opérateurs privés, n'a pas encore été pris en compte dans les études économétriques (voir Nauges et Whittington [2010] pour une revue complète de la littérature). Plus particulièrement, l'analyse des facteurs, qui influencent la demande de raccordement des ménages aux réseaux spontanés développés par les POPs dans des quartiers où le réseau de l'opérateur officiel n'existe pas et où les sources alternatives abondent, s'avère pourtant essentielle pour des décideurs qui viseraient la mise en place de subventions pro-pauvres (subventions aux POPs ou aux ménages). En effet, leur activité palliative, innovante mais exercée aujourd'hui sans autorisation, pourrait intégrer le marché de distribution officiel d'eau, en complément de celui de l'opérateur officiel.

L'objectif de l'article est d'analyser cette demande, à l'aide d'une enquête réalisée en 2009 auprès de 880 ménages dans les quartiers périphériques de Maputo (capitale du Mozambique), plus particulièrement dans les zones d'extension de Maputo, de Matola et de Marracuene. À partir d'une analyse factorielle appliquée aux données de l'enquête, nous avons construit un indice de richesse selon la méthodologie de Filmer et Pritchett [2001] afin d'analyser l'hétérogénéité des quartiers en termes de répartition des revenus selon les quartiles, d'une part, et en termes d'approvisionnement en eau potable des ménages, d'autre part. L'analyse des déterminants de la demande de raccordement des ménages repose sur les résultats de quatre modèles probit. L'article fournit des résultats originaux. En particulier, les résultats des modèles économétriques montrent que la 
localisation des ménages dans certains quartiers est discriminante dans l'accès aux réseaux des POPs (la présence d'un réseau électrique efficace étant nécessaire au développement de leur activité). Par ailleurs, le branchement privé s'avère être l'option privilégiée par les ménages, tant les inconvénients aux sources alternatives abondent. Enfin, bien que la probabilité de raccordement augmente avec l'indice de richesse du ménage, les POPs ne desservent toujours pas les ménages les plus pauvres (ceux qui vivent avec moins d'un dollar par jour et par personne). Ce résultat interroge sur les modalités des politiques de lutte contre la pauvreté à mettre en œuvre (notamment la question de subventionnement des POPs) ; la littérature ayant démontré que certaines politiques de branchements sociaux pouvaient s'avérer contre-redistributives (Brocklehurst, Janssens [2004] pour le cas du Sénégal).

Après avoir présenté les caractéristiques de l'activité des POPs à Maputo, nous commentons la méthodologie et les résultats de l'enquête Ménages en termes de répartition de la richesse selon les quartiers. La section suivante offre un état des lieux de l'approvisionnement en eau des ménages enquêtés selon leurs quartiles. L'analyse économétrique des facteurs explicatifs du choix de raccordement des ménages aux réseaux développés par les POPs est enfin présentée avant de conclure l'étude.

\section{LA SPÉCIFICITÉ DES QUARTIERS PÉRIPHÉRIQUES DE MAPUTO : L'APPROVISIONNEMENT EN EAU POTABLE DES MÉNAGES AUX RÉSEAUX DÉVELOPPÉS PAR DES POPS}

Une série d'études, financées par l'AFD et publiées par Blanc, Cave, Chaponnière [2009] ; Dardenne, Razafinjato, et Dardenne [2008] et Ginisty [2009], révèle des caractéristiques bien particulières au marché de distribution d'eau potable à Maputo dont nous synthétisons, dans cette section, les faits marquants. Le Mozambique a lancé, en 2005, un vaste programme dans sa capitale visant à accroître la production d'eau potable et à étendre le service aux zones périurbaines de l'agglomération formée par Maputo, Matola et Boane. Le service fourni par l'opérateur officiel Aguas de Moçambique (AdeM), qui a signé en 1999 un contrat de type affermage ${ }^{1}$ avec la société de patrimoine FIPAG, est en effet particulièrement dégradé (l'eau est disponible seulement une dizaine d'heures par jour), en particulier dans les zones périurbaines où le réseau n'a souvent pas été étendu. Les performances techniques et commerciales de l'AdeM, qui utilise les eaux superficielles (eaux de rivière) traitées dans une station éloignée du centre ville et à la capacité de production limitée, comptent parmi les plus mauvaises des services d'eau africains. En 2008, le réseau d'adduction d'eau exploité par l'Adem couvre environ $70 \%$ du territoire de la ville de Maputo, mais seuls $33 \%$ de la population consomment l'eau distribuée par l'AdeM. Par ailleurs, les taux de couverture varient considérablement selon les quartiers. Enfin, se pose le problème de l'accès des populations dans les quartiers périphériques en pleine explosion démographique. En effet depuis les années 1990, la croissance

1. Il s'agit d'un contrat de quinze ans signé en 1999 avec la SAUR (qui s'est retirée en 2002). 
urbaine se réalise essentiellement en dehors de la zone de concession de l'AdeM, notamment au nord de Maputo. Face à cette explosion urbaine, les pouvoirs publics n'ont pas développé suffisamment les services de base, dont celui de l'eau potable. C'est pourquoi des fournisseurs informels privés et indépendants (appelés Pequenos Operadores Privados, POPs) se sont lancés dans la réalisation de petits systèmes d'alimentation. En 2008, on dénombre environ 430 POPs à l'initiative de 330 bornes fontaines et de 37500 branchements domiciliaires (connexions à un réseau d'alimentation en eau potable), tandis que l'opérateur officiel AdeM aurait réalisé 300 bornes fontaines et 90000 branchements privés (Blanc, Cave, Chaponnière [2009]). Ces POPs se révèlent donc très dynamiques dans la construction des réseaux d'alimentation dans une cinquantaine de quartiers périphériques de l'agglomération de la capitale, où ils représentent souvent le seul fournisseur d'eau potable (Ginisty [2009]). Aujourd'hui, l'expansion des POPs s'accélère : $86 \%$ des systèmes présents en 2009 ont été créés depuis 2000. Ils se sont d'abord développés dans la périphérie nord de Maputo, l'est de Maputo et l'ouest de Matola. Plus récemment, l'implantation des nouveaux réseaux dépasse la limite administrative des deux municipalités (Maputo et Matola) pour atteindre les quartiers de Marracuene (Nord-Est). Leur développement exclusivement au Nord s'explique par l'absence d'aquifère exploitable au Sud du fait d'une salinité élevée. Nous présentons ci-dessous les caractéristiques du système d'exploitation des réseaux POPs ainsi que les différents services proposés.

\section{Les caractéristiques du système d'exploitation des réseaux POPS}

Les réseaux développés par les POPs reposent essentiellement sur des forages construits grâce à la disponibilité d'une nappe souterraine peu profonde dans la partie nord de l'agglomération, qui, pour diverses raisons ${ }^{2}$, n'a jamais été exploitée par le service public pour alimenter la ville (Dardenne, Razafinjato et Dardenne [2008]). La population des quartiers non desservis par l'AdeM a donc décidé de capter l'eau souterraine à l'aide de petits forages. En effet, le coût d'investissement relativement faible a rendu cette solution préférable à l'attente perpétuelle du réseau de l'opérateur officiel. Une activité de production et de revente de voisinage s'est alors rapidement développée grâce à cette nappe facilement accessible et peu salinisée.

D'un point de vue technique, les systèmes d'alimentation réalisés par les POPs sont pragmatiques mais restent précaires et hydrauliquement peu efficaces. La production est assurée par un ou plusieurs forages installés dans une propriété privée. Le forage principal se situe généralement dans la résidence de l'opérateur qui fait également office de siège technique et administratif de l'activité. L'eau pompée est acheminée vers un réservoir sur tour pour être ensuite distribuée par gravité grâce à un réseau de faible diamètre à peine enterré le long des rues non asphaltées. Souvent, ce réseau débute par quelques conduites individuelles reliant le réservoir à chaque abonné. La multiplication des conduites et l'imbrication

2. Historiquement, l'administration coloniale portugaise a fortement privilégié l'usage des ressources en eau superficielles pour l'alimentation des villes mozambicaines. Même si des études hydrogéologiques avaient débuté dans les années 1980, celles-ci n’ont pu être achevées à cause de l'insécurité régnant dans la région et empêchant la poursuite des travaux de terrain. Enfin, en 2000, le réseau de piézomètres avait été détruit par les inondations. 
de nombreux tuyaux souples forment un réseau appelé « spaghetti ». Toujours du point de vue technique, on relève deux grands types de déficiences en termes de dimensionnement du système et de stockage. Premièrement, le dimensionnement des tours de réservoirs n'a généralement fait l'objet d'aucun calcul préalable, ni pour déterminer la hauteur appropriée, ni pour définir le volume de réservation. C'est pourquoi, quand la pression de distribution de l'eau potable s'avère insuffisante, les opérateurs ajoutent un niveau supplémentaire à la tour de réservoir et d'autres tanks (en plastique). Ce mauvais dimensionnement des systèmes (pertes de charge) nécessite souvent la construction de nouveaux réservoirs quelques mètres plus haut, ce qui affecte le coût d'exploitation des POPs et donc le tarif de l'eau (5 mètres d'exhaure augmente de 10 à $15 \%$ la facture d'électricité, le premier poste de dépense des POPs). Deuxièmement, les réservoirs ne sont vidés et lavés que deux à trois fois par an en moyenne, ce qui pose un problème de qualité de l'eau potable.

\section{Desserte et coût des services fournis par les POPS}

Du point de vue de l'activité de distribution, on distingue trois types de catégories de POPs (voir Snell [1998] pour une typologie plus complète). Certains ont initialement investi dans un forage avec le simple but d'assurer leur propre consommation. Ils ont ensuite développé leur activité pour répondre à la demande des voisins, intéressés à se raccorder à leur système. Cette première catégorie de POPs est plutôt guidée par un objectif social (altruisme, rayonnement personnel au sein de la communauté locale) qu'économique (développement d'une activité professionnelle profitable). C'est pourquoi certains réseaux d'alimentation ont été créés dans un cadre associatif, la taille des systèmes n'ayant pas vocation à grandir. Une seconde catégorie de POPs cherche à s'assurer une rente à partir d'un petit pécule épargné (retraités, travailleurs émigrés revenant au pays après avoir travaillé dans les mines d'Afrique du Sud) qu'ils ont alors choisi d'investir dans la construction d'un forage. Enfin, la dernière génération a plutôt choisi de développer une activité professionnelle dans le but de « gagner sa vie ». Ces POPs, purs entrepreneurs, ont d'abord maintenu en parallèle leur première activité, le temps de disposer de plus grands systèmes organisés autour de plusieurs forages dans des quartiers différents (Dardenne, Razafinjato et Dardenne [2008]). Une étude menée par le FIPAG en $2007^{3}$ estime la population desservie par les POPs (branchements domiciliaires et bornes fontaines incluses) à environ 283000 habitants $^{4}$, le nombre de branchements domiciliaires avoisinant les 40000 (FIPAG [2007]).

La très grande majorité des POPs déclare fournir leurs clients vingt-quatre heures sur vingt-quatre. Néanmoins, les faits révèlent qu'un nombre significatif ferme la nuit pour deux raisons principales. La première raison tient au débit insuffisant de pompage ; les réservoirs étant vides en fin de journée, ils se remplissent le soir et la nuit. La deuxième raison tient à l'absence de personnel d'astreinte en cas de fuites ou de problèmes électromécaniques la nuit.

3. FIPAG/Hydroconseil SSIP Survey, Northern Zone of Greater Maputo, juin 2007

4. Considérant 5,3 habitants par foyer équipé de branchement domiciliaire et 250 bénéficiaires par borne fontaine. 
L'enquête menée en $2007^{5}$ par le FIPAG révèle qu'environ deux tiers des POPs pratiquent un prix au mètre cube (grâce à l'installation de compteurs sur les grands systèmes) et un tiers, une redevance forfaitaire (les petits systèmes ne disposent pas de compteurs). Le tarif au mètre cube est en moyenne de $25 \mathrm{MTN}^{6} /$ $\mathrm{m}^{3}$, tandis que le tarif pratiqué par l'AdeM est de 15,11 MTN $/ \mathrm{m}^{3}$ (FIPAG [2007]). Le prix de $30 \mathrm{MTN} / \mathrm{m}^{3}$ devient cependant de plus en plus fréquent dans les quartiers nouvellement conquis par les POPs et où la concurrence est moins sévère. Les tarifs forfaitaires mensuels (pour ceux qui n'ont pas de compteurs) sont plus variables, puisqu'ils varient entre 20 et $450 \mathrm{MTN} / \mathrm{mois}$ avec une moyenne de $240 \mathrm{MTN} / \mathrm{mois}$, contre une moyenne de $120 \mathrm{MTN} / \mathrm{mois}$ par l'opérateur officiel. Les tarifs pratiqués par les POPs correspondent donc en moyenne au double de ceux pratiqués par l'AdeM. Cependant, dans notre zone d'étude (nous présentons ci-dessous l'échantillon), la concurrence n'existe pas, puisque l'opérateur officiel n'y intervient pas. Les ménages enquêtés ne peuvent donc pas arbitrer entre ces deux options d'approvisionnement (POPs versus AdeM). Enfin, le coût du raccordement diffère fortement selon les POPs et les quartiers, entre 250 MTN et 4000 MTN avec un coût moyen de 1390 MTN. À titre de comparaison, l'AdeM pratique un coût de raccordement de 2300 MTN, mais le matériel de branchement et la pose sont inclus.

Afin d'analyser la demande des ménages envers les POPs, nous avons réalisé, en 2009, une enquête dans les quartiers périphériques de Maputo dont nous présentons ci-dessous la méthodologie et les principaux résultats.

\section{L'ENQUÊTE MÉNAGES RÉALISÉE EN 2009 : ÉCHANTILLONNAGE, INFORMATIONS COLLECTÉES ET RÉPARTITION DE LA RICHESSE DANS LES QUARTIERS PÉRIPHÉRIQUES DE MAPUTO}

Après avoir présenté brièvement la méthodologie retenue pour l'échantillonnage, cette section fournit un descriptif synthétique des informations collectées à partir de l'enquête ainsi que les résultats statistiques relatifs à la répartition des revenus des ménages au sein des quartiers périphériques. Enfin, la dernière sous-section présente une typologie des ménages selon les quartiles de richesse.

\section{Échantillonnage}

L'échantillon de l'enquête, portant sur 880 ménages, a été construit sur la base de la méthodologie du recensement général de la population de 2007 dont nous avons retenu les définitions en termes d'unités. Nous avons fait le choix de ne s'intéresser qu'aux ménages des quartiers périphériques de Maputo, de Matola et de Marracuene qui ne peuvent donc arbitrer entre un réseau POP et le réseau officiel. La méthodologie retenue pour s'assurer de la représentativité de

5. FIPAG/Hydroconseil SSIP Survey, Northern Zone of Greater Maputo, juin 2007.

6. 1 euro est égal à environ 37 MTN (taux de change en juillet 2009). 
l'échantillon repose sur les trois contraintes suivantes : stratification, dispersion et taille. En effet, au lieu de tirer l'échantillon dans la population totale, celuici a été tiré au sein de sous-groupes homogènes de population, identifiés selon des critères géographiques. Trois sous-groupes ont été retenus : Maputo Ville, Maraccuene, Matola (urbain et rural). Pour éviter une très grande dispersion de l'échantillon, nous avons opté pour le sous-groupe homogène des ménages qui habitent dans les quartiers d'intervention des POPs et hors zone desservie par l'AdeM. Enfin, la taille de l'échantillon retenue permet de réaliser des analyses statistique et économétrique (quantitatives). L'enquête a été organisée sous la forme d'un sondage aléatoire à deux degrés. (i) Au premier degré : les unités géographiques (les quarteirãos) sont sélectionnées à partir d'un premier tirage aléatoire dit « aréolaire » (15 quartiers) ; (ii) au second degré : dans chacun des quarteirãos, les ménages sont sélectionnés à l'aide d'un second tirage aléatoire (880 ménages). Le recensement de 2007 dénombre une population totale pour ces trois zones de 294994 personnes, soit 54946 ménages (avec une taille moyenne du ménage de 5,3). Bien que la population urbaine ait augmentée depuis 2007, cette base de référence reste crédible pour déterminer la taille de l'échantillon. La majorité des quartiers enquêtés correspond aux nouveaux quartiers issus du déplacement des populations affectées par les inondations et l'extension de la ville de Maputo. Au final, l'enquête couvre quinze quartiers périphériques appartenant aux trois villes citées précédemment de la province de Maputo : Maputo Ville, Matola, Marracuene. Si les zones de Matola et de Maputo sont urbaines, Marracuene est rurale.

\section{Encadré 1. Les quartiers enquêtés dans les trois villes de l'agglomération de Maputo}

À Maputo Ville : Albazine, Magoanine « A », Magoanine « B », Magoanine « $\mathrm{C} »$, Zimpeto.

À Matola : Khongolote, Ndlavela, $1^{\text {er }}$ de Maio, Kilomètre 15, Nkobe, Matola Gare.

À Marracuene : Abel Jafar, Mateque, Guava, Cumbeza.

Des entretiens qualitatifs, en complément des 880 questionnaires, ont été menés afin de compléter les informations récoltées par l'enquête quantitative et d'apporter une meilleure compréhension des résultats de l'étude. Ces entretiens ont été menés auprès de responsables administratifs (secrétaire de bairro, chefs de quartier), responsables des comités de gestion de l'eau, responsables de groupes de femmes et auprès de quelques POPs. Les entretiens auprès des femmes ont été privilégiés dans la mesure où elles sont très impliquées dans la collecte de l'eau.

\section{Les informations collectées}

La littérature relative aux problématiques d'accès à l'eau potable des populations des Pays en Développement a guidé les choix méthodologiques, dont l'élaboration du questionnaire. D'abord, plusieurs études ont montré que le 
niveau d'éducation et d'alphabétisation (savoir lire ou écrire) des ménages a une influence significative sur le choix du mode d'approvisionnement en eau (Madanat et Humplick [1993] ; Briand, Nauges, Strand et Travers [2010] ; Larson, Minten et Razafindralambo [2006] ; Nauges et Strand [2007] ; Basani, Isham et Reilly [2008] ; Nauges et Van Den Berg [2009]). La composition du ménage affecte également le choix du mode d'approvisionnement en eau ( $\mathrm{Mu}$, Whittington, Briscoe et Barron [1990]). Mu, Whittington, Briscoe et Barron [1990] ont montré que plus le nombre de femmes dans un ménage est élevé, plus la probabilité qu'il soit raccordé au réseau d'adduction d'eau potable est élevée. En effet, la collecte de l'eau aux sources alternatives (borne fontaine ou puits) est principalement effectuée par la main-d'œuvre féminine (Curtis [1986] ; Cairncross et Cliff [1987]). D'autres travaux démontrent que le fait qu'un chef de ménage soit une femme veuve augmente la probabilité que le ménage s'approvisionne à un robinet à domicile (Briand, Nauges et Travers [2009]). Dans ce cas, la femme, qui a en charge les décisions et les dépenses budgétaires familiales, attribue une forte valeur au coût d'opportunité lié au temps alloué à la collecte de l'eau au détriment d'autres activités (Mu, Whittington, Roche [1990]). Les conditions de vie (revenu ou indice de richesse) ainsi que le statut du logement (propriétaire) des ménages affectent également le choix d'approvisionnement au robinet à domicile (Altaf, Whittington, Jamal et Smith [1993] ; Whittington, Lauria et $\mathrm{Mu}$ [1991] ; Briscoe, De Castro, Griffin, North et Olsen [1990] ; Mu, Whittington, Briscoe et Barron [1990] et Ahmad, Bishwanath et Misra [2005] ; Nauges et Van Den Berg [2009] ; Nauges et Strand [2007]). Enfin, la taille du ménage a une influence significative sur la probabilité de choisir le raccordement privé plutôt que les sources alternatives telles que la borne fontaine (Briand, Nauges et Travers [2009]). Les travaux de Nauges et Whittington [2010] ont montré que les différents modes d'approvisionnement en eau représentent souvent des substituts imparfaits.

C'est pourquoi les auteurs recommandent que le questionnaire intègre des informations sur tous les modes alternatifs, qu'ils soient utilisés ou non. Par ailleurs, même si la perception des ménages reste une variable subjective, les auteurs suggèrent de pouvoir comparer les opinions et les satisfactions des ménages sur chaque source et non uniquement sur celle qui est utilisée. Malheureusement, l'enquête que nous avons réalisée à Maputo en 2009 ne permet pas de capter les informations sur l'ensemble des modes d'approvisionnement disponibles. Nous ne connaissons que les opinions des ménages sur la source utilisée. Ceci a limité le choix des variables de décision testées dans les modèles économétriques ci-dessous. Certaines études ont introduit des questions portant sur un ensemble de contraintes liées au mode d'approvisionnement : les heures d'ouverture, les coupures, la pression, la qualité de l'eau (Madanat et Humplick [1993]). Les caractéristiques techniques du service telles que la pression de l'eau influencent fortement la qualité, donc l'attractivité du service. La décision de raccordement des ménages dépend, dans une certaine mesure, de la perception qu'ils ont des petits opérateurs privés d'eau potable (POPs). La partie du questionnaire relative aux différents modes d'approvisionnement en eau utilisés par les ménages comporte une distinction selon les usages dans la mesure où les eaux de boisson et de cuisine ne requièrent pas la même qualité que celle nécessaire aux autres usages (Nauges et Whittington [2010]).

Enfin, le questionnaire d'enquête a été construit de sorte à identifier les caractéristiques socioéconomiques des ménages. La connaissance de leurs conditions 
de vie s'avère très utile dans la mesure où elle permet d'élaborer un indicateur synthétique de richesse. Même si le questionnaire comporte une question sur les revenus des ménages, cette donnée se révèle très souvent biaisée et non objective dans les pays en développement. Le répondant (chef de ménage ou non) ne révèle pas toujours l'ensemble de ses revenus (une partie provenant de l'informel). Filmer et Pritchett [2001] préconisent une méthodologie permettant de mesurer le niveau de richesse d'un ménage. À partir des caractéristiques de l'habitat et des biens d'équipement du ménage, l'analyse factorielle permet de dégager un score représentant un proxy du revenu. Cet indice peut être utilisé comme critère de segmentation des ménages selon une approche matérielle de la richesse et être testé dans l'analyse économétrique pour vérifier son influence dans la décision de raccordement des ménages au réseau des POPs. Un autre critère permet d'identifier les plus pauvres selon une approche plus monétaire : « vivre avec moins d'un dollar par jour et par personne » (ou revenu monétaire par tête inférieur à un dollar, considéré comme critère d' " extrême pauvreté » dans le cadre des Objectifs du Millénaire). Les données de l'enquête nous permettent de tester l'impact de cette variable dans la demande de raccordement aux POPs des ménages. Ces deux approches de la richesse, l'une matérielle et l'autre monétaire, sont alors testées dans les modèles.

\section{Construction de l'indice de richesse}

La première étape consiste à choisir les différentes variables à utiliser pour construire l'indice synthétique de richesse. L'objectif est de retenir le maximum de variables afin d'augmenter la variation interindividuelle de la valeur de l'indice et d'obtenir une distribution plus régulière des ménages selon les quartiles. Initialement, à partir des données de l'enquête 2009, trente-trois variables ont été retenues ${ }^{7}$ et recodifiées afin d'être transformées en variables binaires de type « oui » ou « non », c'est-à-dire « 1 » si oui et « $0 »$ si non (voir le tableau A1 en annexe qui détaille les variables constitutives de l'indice). La réalisation d'un test de corrélation a débouché sur l'élimination de neuf variables ${ }^{8}$, ce qui maintient vingt-quatre variables non corrélées pour l'analyse factorielle?

Dans une seconde étape, nous procédons à une analyse factorielle pour calculer l'indice de richesse. La méthode consiste à appliquer un poids correspondant au score factoriel de chaque bien sur le premier axe factoriel. Le score ou le poids d'un ménage $i$, selon qu'il possède un bien $j$, est déterminé selon la méthodologie présentée dans le tableau A2 (annexe). Le tableau A3 présente, pour chaque variable, les données relatives au calcul de l'indice (score) de chaque ménage selon qu'il possède ou non chacun des biens.

7. Les caractéristiques de l'habitat du ménage : le matériau dominant du toit, du sol, du mur, le type de sanitaire, le type d'habitat, le mode d'évacuation des eaux usées, la disposition d'un bain/ douche à l'intérieur, les biens d'équipement du ménage tels que télévision, radio, voiture, vélo, téléphone, etc.

8. Les neuf variables éliminées sont : le ménage possède-t-il « un bain/douche avec eau courante », " un WC moderne ", « un fer à repasser électrique », " une télévision », « une maison moderne », « un habitat précaire », « un mur en ciment», « un toit en zinc », et « un sol en ciment»?

9. Seules les variables les plus discriminantes sont retenues : si deux variables (biens) sont corrélées, la moins discriminante (celle qui était possédée majoritairement par les ménages) est éliminée. 
Enfin, dans une dernière étape, les indices de richesse des différents ménages sont classés par ordre croissant afin d'établir une segmentation par quartile. L'échantillon est donc divisé en quatre sous-groupes d'effectifs égaux de 220 ménages. Le premier quartile (IR1) correspond aux $25 \%$ « les plus pauvres », le second quartile (IR2) aux $25 \%$ de « modestes », le troisième quartile (IR3) renvoie aux $25 \%$ d' " aisés » et le dernier quartile (IR4) aux $25 \%$ appelés « très riches $»$.

\section{Répartition de la richesse dans les quartiers périphériques}

Les trois agglomérations étudiées présentent des caractéristiques différentes. Par ailleurs, au sein de chaque ville, les quartiers ne sont pas homogènes. Maputo Ville présente la plus forte proportion de ménages riches $(64 \%)$ relativement à Matola (39\%) et à Marracuene (35\%).

\section{Maputo}

Maputo est une ville où la grande majorité des ménages sont riches $(64 \%$ des ménages des IR3 et IR4). $33 \%$ appartiennent aux $25 \%$ les plus riches (IR4) et $31 \%$ correspondent aux ménages aisés (IR3). Maputo Ville comporte seulement $15 \%$ de ménages très pauvres (IR1). Ces résultats ne sont pas surprenants puisque cette ville est la capitale de la province de Maputo. Elle comprend des quartiers très riches et bien structurés.

Bien que Maputo soit la ville la plus riche, elle cache des disparités selon les quartiers ( $c f$. graphique A1 en annexe). Le quartier Magoanine « B » est le plus nanti avec la moitié des ménages appartenant à la classe des très riches (IR4) ; Zimpeto et Magoanine «A » représentent des quartiers relativement aisés. En revanche, le quartier Albazine comporte $45 \%$ de ménages pauvres ; $28 \%$ appartiennent à la classe des très pauvres (IR1) et $17 \%$ à la classe des modestes (IR2). Enfin, le quartier Magoanine « $\mathrm{C} »$ rassemble le plus de ménages très pauvres (plus de $20 \%$ appartiennent à l'IR1).

\section{Matola}

Matola est une ville où plus de la moitié $(51 \%)$ de la population est pauvre (IR1 et IR2 confondus). $35 \%$ des ménages appartiennent aux $25 \%$ les plus pauvres (IR1) et $26 \%$ aux modestes (IR2). Le pourcentage de ménages très riches est faible ; il est de l'ordre de $17 \%$, soit deux fois moins qu'à Maputo.

Les quartiers de Matola Gare, de Kilomètre 15 et de Nkobe rassemblent le plus de ménages pauvres avec respectivement $82 \%, 80 \%$ et $79 \%$ (IR1 et IR2 confondus) ( $c f$. graphique A2). Parmi ces trois quartiers, Matola Gare représente celui où la part des ménages aisés ( $8 \%$ de la classe IR3) et celle des très riches ( $7 \%$ de la classe IR4) sont les plus faibles. Ces quartiers sont également les plus touchés par les problèmes d'accès à l'électricité et à l'eau potable ( $c f$. graphique A3). Le quartier de Ndlavela correspond au quartier le plus riche de Matola avec plus de $40 \%$ de ménages aisés (IR3) et $23 \%$ de ménages très riches (IR4). 


\section{Marracuene}

Marracuene est la ville où l'on trouve le plus de ménages pauvres (environ $65 \%$ des ménages, IR1 et IR2 confondus ; $c f$. graphique A4). Mais c'est aussi la seconde ville après Maputo où l'on observe le plus de ménages riches $(21 \%$ des ménages appartiennent au groupe IR4). La grande caractéristique de Marracuene tient à sa ruralité. De nombreux habitants ont migré (principalement d'Afrique du Sud) et disposent de niveaux de vie supérieurs à la moyenne. Quant aux autres, la plupart occupent des emplois précaires (domestique, agent de sécurité). Cumbeza représente le quartier le plus riche (presque $45 \%$ de très riches, IR4) et Habel Jafar le plus pauvre (38\% de ménages très pauvres (IR1) et $55 \%$ de ménages modestes (IR2)). Les quartiers de Mateque et de Guava sont plus hétérogènes mais, en moyenne, les ménages pauvres et modestes sont plus nombreux que les ménages aisés et très riches.

\section{Typologie des ménages selon les indices de richesse}

À partir des résultats statistiques des données de l'enquête, nous présentons maintenant les caractéristiques socioéconomiques des ménages segmentés selon les quartiles de richesse.

\section{Les ménages pauvres (IR1)}

La majeure partie des ménages du premier quartile vivent à Matola (plus du tiers) et plus particulièrement à Matola Gare $(60 \%)$. Majoritairement féminins (65\%), divorcés ou veufs, les chefs de ménage pauvres n'ont pas dépassé le niveau primaire (69 \%) et occupent un emploi d'agriculteur ou de maraîcher. Les femmes, chefs de ménage, sont majoritairement des célibataires, séparées/divorcées ou veuves ; leur situation économique est généralement précaire (revenus faibles). Lors d'un entretien, une enquêtée a révélé :

« Je suis veuve ; je ne travaille pas et la vie est très dure ; je dois me débrouiller seule avec mes enfants ; on a du mal à joindre les deux bouts. »

Il s'agit également du quartile qui rassemble le plus d'occupants à titre gratuit de leur logement (63\% de tous les occupants à titre gratuit).

\section{Les ménages modestes (IR2)}

Plus du tiers des ménages modestes vivent à Marracuene, plus particulièrement à Habel Jafar (50\%). Ils sont presque tous propriétaires de leurs logements $(93 \%)$. Les chefs de ménage sont autant représentés par les femmes que par les hommes. Ils sont généralement agriculteurs ou inactifs et ont, pour la plupart, un faible niveau d'instruction ( $58 \%$ n'ont pas dépassé le niveau primaire).

\section{Les ménages aisés (IR3)}

Près du tiers des ménages aisés habitent à Maputo, plus particulièrement à Magoanine « $\mathrm{A}$ » et à Zimpeto (respectivement $34 \%$ et $32 \%$ ). Les chefs de ménage sont majoritairement masculins. Plus de la moitié ont dépassé le niveau 
d'instruction primaire ( $52 \%$ ). Ils sont quasiment tous propriétaires de leur logement $(93 \%)$.

\section{Les ménages riches (IR4)}

Le tiers des ménages riches vit à Maputo, plus particulièrement à Magoanine « B » $(49 \%)$. Une part importante de ces ménages vit dans des logements de fonction. D'un niveau d'éducation secondaire ou universitaire (73\%), les chefs de ménage sont le plus souvent des hommes mariés et entrepreneurs.

L'analyse statistique présentée ci-dessus montre que la construction de l'indice de richesse s'avère pertinente puisque les quartiers périphériques de Maputo se révèlent hétérogènes du point de vue de la répartition de la richesse ; les ménages présentant des caractéristiques socioéconomiques différentes selon leur classe d'appartenance aux quartiles. La section suivante présente alors un état des lieux de l'approvisionnement en eau potable des ménages selon ces quartiles.

\section{L'APPROVISIONNEMENT EN EAU POTABLE DES MÉNAGES : UNE ANALYSE SELON LES QUARTILES DE RICHESSE}

Nous présentons les résultats statistiques relatifs aux différents modes d'approvisionnement en eau des ménages sur l'ensemble de la zone enquêtée puis selon les quartiles.

\section{Résultats globaux sur la zone d'étude}

De manière intuitive, les résultats illustrés dans le tableau 1 montrent que les ménages raccordés aux réseaux des POPs consomment relativement plus que les ménages non raccordés : en moyenne 56 litres par jour et par personne (contre respectivement 29, 32 et 31 litres par jour et par personne aux bornes fontaines, puits et sources alternatives). Contrairement à ce que l'on peut observer dans d'autres pays d'Afrique (Collignon et Vezina [2000]), le prix moyen de l'eau au mètre cube est plus élevé aux branchements domiciliaires (25 MTN) qu'aux bornes fontaines (19 MTN). Cette situation s'explique par la non-régulation de l'activité des POPS.

Tableau 1. Approvisionnement en eau potable des ménages raccordés et non raccordés

\begin{tabular}{c|c|c|c|c}
\hline & Raccordés & \multicolumn{3}{|c}{ Non raccordés } \\
\cline { 3 - 5 } & & $\begin{array}{c}\text { Borne } \\
\text { fontaine }\end{array}$ & Puits & $\begin{array}{c}\text { Autres sources } \\
\text { (voisin et forages) }\end{array}$ \\
\hline Part de 1'échantillon & $54 \%$ & $23 \%$ & $10 \%$ & $13 \%$ \\
Consommation en 1/j/p & 56 & 29 & 32 & 31 \\
Prix en MTN & 25 & 19 & 8 & $6-36$ \\
Distance à parcourir & 0 & $243 \mathrm{~m}$ & $114 \mathrm{~m}$ & \\
Temps de collecte & 0 & $67 \mathrm{~min}$ & $59 \mathrm{~min}$ & \\
\hline
\end{tabular}

Source : Calculs des auteurs. 
Si $54 \%$ des ménages de la zone enquêtée ont accès aux réseaux des POPs, on observe des disparités selon les quartiers périphériques. En particulier, les quartiers de Cumbeza et de Magoanine « $\mathrm{A}$ » et « $\mathrm{B}$ » sont relativement bien dotés en branchements privés (plus de $70 \%$ des ménages). À l'inverse, dans les quartiers de Matola Gare, de Guava et de Nkobe, moins de $40 \%$ des ménages bénéficient de l'accès aux réseaux d'eau potable (graphique $\mathrm{A} 4$, annexe).

Comme indiqué dans le tableau 1, $46 \%$ des ménages ne sont pas raccordés aux réseaux d'eau potable développés par les POPs. Ne bénéficiant pas des branchements domiciliaires, ces ménages utilisent, par défaut, d'autres modes d'approvisionnement tels que les bornes fontaines (23\%), les puits $(10 \%)$ ou, encore, l'eau de voisinage et des forages ( $13 \%$ pour ces deux derniers). Interrogés sur leurs attentes, les ménages non raccordés sont ceux qui souhaitent le plus « être connectés à un réseau POP » ou « avoir de l'eau courante à la maison ».

Sur l'ensemble de l'échantillon, près d'un quart des ménages s'approvisionne auprès des bornes fontaines. Certaines, financées par des ONG, sont gratuites. Une part relativement importante se révèle insatisfait du service, la pénibilité du portage de l'eau sur les distances jugées longues apparaissant comme la principale source d'insatisfaction. Dans certains quartiers, les ménages se plaignent également du goût jugé trop salé de l'eau.

Les $10 \%$ de ménages utilisateurs des puits parcourent une distance relativement plus faible (114 mètres) que celle aux bornes fontaines (243 mètres). En effet, les puits sont relativement plus nombreux que ces dernières et sont le plus souvent directement localisés dans les concessions ou auprès des voisins. L'eau des puits est généralement gratuite mais, dans certains quartiers (Kilomètre 15, Matola Gare...) délaissés (absence de réseau électrique), l'eau du puits est vendue ( 8 MTN en moyenne le mètre cube). Les utilisateurs sont conscients des risques sanitaires liés aux dépôts. Un enquêté déclare :

« L'eau du puits n'est pas protégé, il y a des organismes vivants dedans. Il y a des crapauds dans le puits, mais on n'a pas le choix. »

Enfin, $13 \%$ des ménages utilisent essentiellement des sources alternatives telles que l'eau de voisinage et les forages publics. Les ménages recourant à l'eau de voisinage sont ceux qui paient en moyenne le plus cher l'eau (36 MTN le $\mathrm{m}^{3}$ ). À l'inverse, les utilisateurs des forages sont ceux qui la paient le moins cher (6 MTN le $\left.\mathrm{m}^{3}\right)$. Rappelons que les forages, principalement réalisés par les ONG, ne facturent qu'un montant forfaitaire correspondant à une partie du coût de l'entretien. Cette source regroupe le plus d'insatisfaits, bien que l'eau y soit quasi gratuite. Le secrétaire de bairro de Khongolote déclare :

« Les forages sont l'œuvre d'ONG (Croix-Rouge, par exemple). Son eau est payée de façon symbolique. Le système n'est pas efficace car les ventes ne couvrent pas les coûts de fonctionnement, de sorte qu'une fois qu'ils sont en panne, ils sont laissés à l'abandon. Les populations n'aiment pas du tout l'eau des forages. » 


\section{Approvisionnement en eau potable des ménages selon les quartiles de richesse}

L'analyse des modes d'approvisionnement en eau selon les quartiles de richesse montre clairement que plus les ménages sont pauvres, moins ils sont raccordés aux réseaux développés par les POPs (tableau 2). $79 \%$ des ménages pauvres et $57 \%$ des ménages modestes ne bénéficient pas de branchements privés. En revanche, la plupart des ménages aisés et riches sont raccordés (70\% et $86 \%$ ). Par ailleurs, les quantités d'eau consommées augmentent avec le niveau de richesse. Si les ménages pauvres et modestes ne consomment que 33 et 39 litres d'eau par jour et par personne, les ménages aisés et riches en consomment 43 et plus de 60 litres par jour et par personne.

Le tableau 2 illustre les spécificités de l'approvisionnement en eau potable des quatre classes de ménages.

Tableau 2. Approvisionnement en eau potable des non-raccordés selon l'indice de richesse

\begin{tabular}{|c|c|c|c|c|c|c|c|}
\hline $\begin{array}{c}\text { Classes de } \\
\text { ménages } \\
\text { selon l'indice } \\
\text { de richesse }\end{array}$ & $\begin{array}{c}\text { Part des } \\
\text { ménages } \\
\text { non } \\
\text { raccordés } \\
\text { au réseau }\end{array}$ & $\begin{array}{c}\text { Sources } \\
\text { d'approvision- } \\
\text { nement en eau }\end{array}$ & $\begin{array}{l}\text { Répartition des non- } \\
\text { raccordés selon les } \\
\text { sources d'approvi- } \\
\text { sionnement en eau } \\
\text { utilisées (en \%) }\end{array}$ & $\begin{array}{c}\text { Consommation } \\
1 / \mathrm{j} / \text { personne }\end{array}$ & $\begin{array}{c}\text { Prix de } \\
\text { l'eau au } \\
\mathrm{m}^{3} \text { en } \\
\text { MTN }\end{array}$ & $\begin{array}{c}\text { Distance } \\
\text { à } \\
\text { parcourir }\end{array}$ & $\begin{array}{c}\text { Temps } \\
\text { de } \\
\text { collecte }\end{array}$ \\
\hline $\begin{array}{c}\text { IR1 } \\
\text { «Pauvres » }\end{array}$ & $79 \%$ & $\begin{array}{c}\text { Borne fontaine } \\
\text { Puits } \\
\text { Voisin } \\
\text { Forages }\end{array}$ & $\begin{array}{c}41 \\
16 \\
18 \\
4\end{array}$ & $\begin{array}{l}26 \\
29 \\
28 \\
33\end{array}$ & $\begin{array}{c}18 \\
10 \\
39 \\
0\end{array}$ & $\begin{array}{l}250 \mathrm{~m} \\
187 \mathrm{~m} \\
97 \mathrm{~m} \\
78 \mathrm{~m}\end{array}$ & $\begin{array}{l}78 \mathrm{~min} \\
67 \mathrm{~min} \\
44 \mathrm{~min} \\
27 \mathrm{~min}\end{array}$ \\
\hline $\begin{array}{c}\text { IR2 } \\
\text { « Modestes » }\end{array}$ & $57 \%$ & $\begin{array}{c}\text { Borne fontaine } \\
\text { Puits } \\
\text { Voisin } \\
\text { Forages } \\
\end{array}$ & $\begin{array}{c}28 \\
13 \\
11 \\
5 \\
\end{array}$ & $\begin{array}{l}35 \\
33 \\
32 \\
27 \\
\end{array}$ & $\begin{array}{c}19 \\
7 \\
32 \\
0 \\
\end{array}$ & $\begin{array}{c}250 \mathrm{~m} \\
65 \mathrm{~m} \\
72 \mathrm{~m} \\
57 \mathrm{~m} \\
\end{array}$ & $\begin{array}{l}59 \mathrm{~min} \\
67 \mathrm{~min} \\
28 \mathrm{~min} \\
30 \mathrm{~min} \\
\end{array}$ \\
\hline $\begin{array}{c}\text { IR3 } \\
\text { « Aisés » }\end{array}$ & $30 \%$ & $\begin{array}{c}\text { Borne fontaine } \\
\text { Puits } \\
\text { Voisin }\end{array}$ & $\begin{array}{c}15 \\
9 \\
6 \\
\end{array}$ & $\begin{array}{l}28 \\
40 \\
33 \\
\end{array}$ & $\begin{array}{l}19 \\
37 \\
31 \\
\end{array}$ & $\begin{array}{l}165 \mathrm{~m} \\
80 \mathrm{~m} \\
11 \mathrm{~m} \\
\end{array}$ & $\begin{array}{l}63 \mathrm{~min} \\
48 \mathrm{~min} \\
37 \mathrm{~min} \\
\end{array}$ \\
\hline $\begin{array}{c}\text { IR4 } \\
\text { « Riches » }\end{array}$ & $14 \%$ & $\begin{array}{c}\text { Borne fontaine } \\
\text { Puits } \\
\text { Voisin } \\
\text { Forages }\end{array}$ & $\begin{array}{l}5 \\
4 \\
3 \\
2\end{array}$ & $\begin{array}{l}30 \\
48 \\
41 \\
44\end{array}$ & $\begin{array}{c}17 \\
5 \\
24 \\
0\end{array}$ & $\begin{array}{c}284 \mathrm{~m} \\
148 \mathrm{~m} \\
44 \mathrm{~m} \\
215 \mathrm{~m}\end{array}$ & $\begin{array}{l}55 \mathrm{~min} \\
30 \mathrm{~min} \\
34 \mathrm{~min} \\
73 \mathrm{~min}\end{array}$ \\
\hline
\end{tabular}

Source : Calculs des auteurs.

Les ménages pauvres et modestes étant majoritairement non connectés aux réseaux des POPs, nous mettons l'accent, dans nos commentaires, sur leurs consommations aux autres sources d'approvisionnement en eau.

Dans la zone enquêtée, $79 \%$ des ménages pauvres ne sont pas raccordés. Parmi eux, $41 \%$ (soit $52 \%$ des non-raccordés du groupe) s'approvisionnent à la borne fontaine (c'est la source la plus utilisée). Les deux autres principaux modes d'alimentation en eau sont les puits (16\%) et les voisins (18\%). Il est intéressant de noter que les forages publics qui délivrent une eau gratuite sont très peu utilisés. La consommation aux bornes fontaines est relativement plus faible que celle aux puits et au voisinage (26 litres par jour et par personne contre respectivement 29 litres par jour et par personne et 28 litres par jour et par personne). Le prix de l'eau aux bornes fontaines (18 MTN par $\mathrm{m}^{3}$ ) est plus faible que celui facturé par le 
voisinage (39 MTN le $\mathrm{m}^{3}$ ), mais cette alternative permet de diminuer la distance. En effet, les ménages pauvres qui recourent aux bornes fontaines doivent parcourir en moyenne 250 mètres contre 97 mètres pour accéder à l'eau du voisinage. L'eau des puits est moins coûteuse (10 MTN le $\left.\mathrm{m}^{3}\right)$ mais nécessite de parcourir 187 mètres en moyenne. De nombreux puits fournissent une eau gratuite sauf dans les quartiers marqués par une offre très limitée (Kilomètre 15 et Matola Gare). Les ménages pauvres insatisfaits représentent $46 \%$ des utilisateurs des bornes fontaines, $59 \%$ des utilisateurs des puits et $42 \%$ de ceux qui recourent à l'eau de voisinage. Les principaux motifs d'insatisfaction sont la pénibilité du portage de l'eau pour un approvisionnement aux bornes fontaines, la qualité de l'eau (turbidité, potabilité et risques de maladies) pour ceux qui utilisent les puits, le prix jugé trop élevé pour ceux qui achètent l'eau auprès de leurs voisins et, enfin, l'odeur de l'eau aux forages publics.

$28 \%$ des ménages modestes (soit $49 \%$ des non-raccordés du groupe) s'approvisionnent à la borne fontaine avec une consommation moyenne de 35 litres par jour et par personne, soit 9 litres de plus que la consommation moyenne des ménages pauvres à cette même source. $13 \%$ des ménages modestes (soit $23 \%$ des non-raccordés du groupe) utilisent l'eau du puits. Les puits restent relativement plus proches (une distance de 65 mètres en moyenne) que les autres modes d'approvisionnement (forages exclus) et le prix relativement plus faible. L'achat d'eau auprès des voisins concerne $11 \%$ des ménages modestes (soit $19 \%$ des non-raccordés du groupe) avec un prix relativement plus élevé qu'aux autres modes. Enfin, $5 \%$ (soit $9 \%$ des ménages modestes non raccordés) utilisent l'eau des forages publics. On retrouve approximativement les mêmes raisons d'insatisfaction aux différentes sources (dans des proportions proches) que celles observées chez les pauvres.

\section{MODÉLISATION DES DÉTERMINANTS DU RACCORDEMENT DES MÉNAGES AUX RÉSEAUX POP D’EAU POTABLE}

Nous estimons un modèle qui vise à étudier la probabilité que les ménages choisissent de se raccorder aux branchements domiciliaires des POPs. Ce modèle permet de mettre en évidence les déterminants socioéconomiques de la demande de connexion des ménages aux réseaux issus des opérateurs indépendants.

\section{Spécification du modèle et les variables d'analyse}

Nous cherchons à identifier les facteurs qui influencent la décision de raccordement des ménages aux réseaux développés par les POPs dans les quartiers périphériques de Maputo où seules les sources alternatives (bornes fontaines ${ }^{10}$, puits, voisins, forages) existent, le réseau de l'opérateur officiel (AdeM) n'intervenant pas. Il n'y a donc pas d'arbitrage entre un réseau POP et le réseau AdeM. les POPS.

10. Les bornes fontaines implantées dans la zone de notre étude sont toutes développées par 
Dans ces conditions, face à une variable dépendante qualitative et des variables explicatives, à la fois qualitatives et quantitatives, nous optons pour l'utilisation d'un modèle probit.

Ce modèle peut être formalisé de la façon suivante :

$$
\begin{aligned}
& y_{i}^{*}=\beta_{0}+\beta_{i} X_{i}+\mu_{i} \\
& y_{i}=1 \text { si } y_{i}^{*}>0 \text { ou } y_{i}=1 \text { si } \beta_{0}+\beta_{i} X_{i}+\mu_{i}>0 \text { et } y_{i}=0 \text { autrement }
\end{aligned}
$$

avec :

$\mu_{i}$ est le terme d'erreur qui suit une loi normale, $\beta_{i}$ sont les coefficients des variables explicatives,

$y_{i}^{*}$ est une variable latente (inobservable) même si on peut observer le comportement des individus.

La variable dépendante $y_{i}$ représente la probabilité qu'un ménage des quartiers périphériques de Maputo soit raccordé à un réseau d'eau potable POP. Cette variable prend la valeur « 1 » si le ménage est raccordé ou « 0 » s'il ne l'est pas.

Le choix des variables pertinentes à retenir s'inspire de la revue de la littérature, présentée précédemment, sur l'analyse du comportement des ménages face à l'eau ${ }^{11}$ (voir également Nauges et Whittington [2010] pour une revue plus complète). Par ailleurs, seules des variables non corrélées peuvent être maintenues dans le modèle. Pour confirmer l'absence de multicolinéarité, deux méthodes sont retenues. D'abord, la corrélation deux à deux entre les variables est calculée et conduit à la suppression de celles pour lesquelles le coefficient est supérieur à 0,50 . Puis le test de multicolinéarité VIF (variance inflation factor) a été réalisé (Cahuzac et Bontemps [2008] ${ }^{12}$; voir résultats dans le tableau A4 en annexe).

Pour éviter le problème d'endogénéité de la variable d'opinion ${ }^{13}$ des « ménages branchés insatisfaits du service d'eau ", liée à sa subjectivité, nous attribuons pour chaque ménage (observation) la valeur correspondant à la part des ménages insatisfaits à l'échelle du quartier (Briand, Nauges et Travers [2009]). Un test d'endogénéité de la variable « indice de richesse » a été réalisé ( $c f$. tableau A5). L'instrumentalisation de l'indice de richesse ne modifie pas les conclusions du modèle.

Les variables correspondant à l' « âge » du chef de ménage, la « taille » du ménage, l' « indice de richesse » du ménage et « la part des branchés insatisfaits du service dans le quartier » sont quantitatives, tandis que toutes les autres variables sont transformées en variables binaires. Le fait que le chef de ménage soit une « femme », soit « locataire » de son logement, soit « analphabète », « vive avec

11. Nous avons tenté de tester les variables de prix et de qualité selon les sources même si le questionnaire d'enquête ne permettait pas d'avoir ces informations pour chacune des sources. En effet, les ménages n'ont répondu que pour la principale source utilisée. Nous avons alors reconstitué les données manquantes en calculant des moyennes par quartier pour les réattribuer à chaque ménage. Les résultats ne sont pas intéressants (les variables n'apparaissent pas significatives). De plus, cela introduit de la multicolinéarité.

12. «Pour détecter les risques de colinéarité, il suffit de calculer une mesure du changement de la variance de chacun des facteurs lorsqu'on les introduit dans la régression. Cette mesure est souvent notée VIF pour variance inflation factor. » (Cahuzac et Bontemps [2008].)

13. De nombreuses autres variables d'opinion ont été testées dans le modèle. Toujours non significatives, ces variables n'ont pas été conservées. 
moins d'un dollar par jour et par personne $»^{14}$ prennent la valeur 1 sinon 0 . Le modèle intègre également treize variables dummy " quartier » construites en prenant comme référence le quartier Habel Jafar, le quartier Zimpeto ayant été supprimé du modèle (colinéarité).

Finalement, le modèle est le suivant :

$Y=$ fonction [Le chef de ménage est une femme $\left(X_{1}\right)+$ L'âge du chef de ménage $\left(\mathrm{X}_{2}\right)+$ Le chef de ménage est analphabète $\left(\mathrm{X}_{3}\right)+$ Le ménage est locataire de son logement $\left(\mathrm{X}_{4}\right)+$ La taille du ménage $\left(\mathrm{X}_{5}\right)+$ L'indice de richesse du ménage $\left(\mathrm{X}_{6}\right)+$ Le ménage vit avec moins d'un dollar par jour et par personne $\left(\mathrm{X}_{7}\right)+$ La part des branchés insatisfaits du service dans le quartier $\left(\mathrm{X}_{8}\right)+13$ variables dummy « quartier » $\left(\mathrm{X}_{9}\right.$ à $\left.\left.\mathrm{X}_{21}\right)+\mu_{i}\right]$.

Le tableau A6 présente les statistiques descriptives, par quartier, des différentes variables explicatives du modèle. Les quartiers Matola et Albazine comptent le plus de femmes chefs de ménage ( $88 \%$ et $84 \%$ ). Guava dispose relativement moins de locataires que les autres quartiers (88\%). On observe une distribution de la taille des ménages relativement homogène selon les quartiers (entre 4 et 6). L'indice de richesse est le plus élevé à Habel Jafar et le plus faible à Albazine. Plus des deux tiers des ménages vivent avec moins d'un dollar par jour et par personne (69\%) à Magoanine « $\mathrm{B} »$, contre un tiers seulement à Habel Jafar. La part des ménages raccordés aux réseaux des POPs insatisfaits du service d'eau est la plus élevée à Habel Jafar (23 \%).

\section{Résultats du modèle et tests de robustesse}

Les résultats du modèle probit, intégrant à la fois l'indice de richesse et le fait de vivre avec moins d'un dollar par jour et par personne, sont présentés dans le tableau 3 ci-dessous. La régression a été réalisée sur un échantillon de 871 ménages (479 raccordés aux réseaux d'eau potable et 392 non raccordés) de la zone enquêtée. Sur les 479 ménages connectés, 376 ont été correctement prédits (avec une probabilité supérieure à 0,5\%). Sur les 392 ménages non connectés, 282 ont été correctement prédits. Le modèle est globalement satisfaisant car il permet de prédire correctement $75,55 \%$ des cas. Par ailleurs, le $\mathrm{R}^{2}$ de Mac Fadden est égal à 29,6\%, ce qui représente une valeur supérieure au seuil jugé minimal pour justifier la robustesse d'un modèle probit $(25 \%)$.

Le fait que le chef de ménage soit analphabète diminue la probabilité qu'il soit raccordé à un branchement privé POP. La littérature a démontré que la probabilité d'être connecté augmente avec le niveau d'instruction, et cela pour plusieurs raisons. Dans certains cas, le revenu peut augmenter avec le niveau d'instruction. Par ailleurs, le fait de ne savoir ni lire ni écrire limite les opportunités d'accès à l'information. Un faible niveau d'instruction limite la compréhension des enjeux liés au fait de disposer de l'eau potable chez soi : intérêt sanitaire, facilité de collecte, disponibilité constante et gain en termes de temps (le coût d'opportunité du temps augmentant avec le niveau d'instruction). Les statistiques descriptives révèlent que le taux de raccordement aux réseaux des POPs n'est que de $27 \%$ parmi les ménages dont le chef ne sait ni lire ni écrire, tandis qu'il atteint $46 \%$

14. Il s'agit du revenu par tête inférieur à un dollar qui est considéré par la Banque mondiale, dans le cadre des Objectifs du Millénaire, comme le critère de mesure de l'extrême pauvreté. 
Tableau 3. Estimation de la probabilité d'être branché à un réseau POP avec un modèle probit comportant à la fois l'indice de richesse et le fait de vivre avec moins de un dollar par jour et par personne

\begin{tabular}{|c|c|c|c|c|c|c|}
\hline \multicolumn{7}{|c|}{ Variable dépendante : « Probabilité pour un ménage d'être branché à un réseau POP » } \\
\hline Variables explicatives & Coef. & Std. Err. & $\mathrm{P}>z$ & $d y / d x$ & Std. Err. & $\mathrm{X}$ \\
\hline Le chef de ménage est une femme (1/0) & 0,056 & 0,133 & 0,675 & 0,022 & 0,052 & 0,219 \\
\hline L’âge du chef de ménage & $-0,004$ & 0,005 & 0,482 & $-0,001$ & 0,002 & 40,364 \\
\hline Le chef de ménage est analphabète $(1 / 0)$ & $-0,373 * *$ & 0,205 & 0,068 & $-0,148$ & 0,081 & 0,083 \\
\hline $\begin{array}{l}\text { Le ménage est locataire de son logement } \\
(1 / 0)\end{array}$ & $-0,588 * * *$ & 0,276 & 0,033 & $-0,231$ & 0,103 & 0,045 \\
\hline La taille du ménage & 0,034 & 0,026 & 0,181 & 0,013 & 0,010 & 5,204 \\
\hline L'indice de richesse du ménage & $0,167 * * * *$ & 0,017 & 0,000 & 0,066 & 0,007 & 0,010 \\
\hline $\begin{array}{l}\text { Le ménage vit avec moins d'un dollar } \\
\text { par jour et par personne }\end{array}$ & $-0,347 * * * *$ & 0,115 & 0,003 & $-0,136$ & 0,045 & 0,452 \\
\hline $\begin{array}{l}\text { La part des branchés insatisfaits du } \\
\text { service dans le quartier }\end{array}$ & $0,043 * * * *$ & 0,015 & 0,006 & 0,017 & 0,006 & 8,892 \\
\hline $\begin{array}{l}\text { Le ménage habite dans le quartier } \\
\text { Matèque }\end{array}$ & $1,615 * * * *$ & 0,39 & 0,000 & 0,400 & 0,038 & 0,024 \\
\hline $\begin{array}{l}\text { Le ménage habite dans le quartier } \\
\text { Guava }\end{array}$ & $-0,639 * * *$ & 0,311 & 0,040 & $-0,250$ & 0,114 & 0,047 \\
\hline $\begin{array}{l}\text { Le ménage habite dans le quartier } \\
\text { Matola Gare }\end{array}$ & $-0,572 *$ & 0,36 & 0,112 & $-0,225$ & 0,136 & 0,067 \\
\hline $\begin{array}{l}\text { Le ménage habite dans le quartier } \\
\text { Cumbeza }\end{array}$ & $0,993 * * * *$ & 0,315 & 0,002 & 0,314 & 0,069 & 0,046 \\
\hline $\begin{array}{l}\text { Le ménage habite dans le quartier } \\
\text { Nkobe }\end{array}$ & $-0,096$ & 0,251 & 0,701 & $-0,038$ & 0,099 & 0,046 \\
\hline $\begin{array}{l}\text { Le ménage habite dans le quartier } \\
\text { Kilomètre } 15\end{array}$ & 0,12 & 0,286 & 0,675 & 0,046 & 0,109 & 0,046 \\
\hline $\begin{array}{l}\text { Le ménage habite dans le quartier } \\
1^{\text {er }} \text { de Maio }\end{array}$ & $0,792 * * * *$ & 0,238 & 0,001 & 0,269 & 0,064 & 0,069 \\
\hline $\begin{array}{l}\text { Le ménage habite dans le quartier } \\
\text { Ndlavela }\end{array}$ & $0,738 * * * *$ & 0,237 & 0,002 & 0,256 & 0,068 & 0,088 \\
\hline $\begin{array}{l}\text { Le ménage habite dans le quartier } \\
\text { Khongolote }\end{array}$ & $0,816^{* * * *}$ & 0,302 & 0,007 & 0,275 & 0,079 & 0,068 \\
\hline $\begin{array}{l}\text { Le ménage habite dans le quartier } \\
\text { Magoanine « } \mathrm{A} »\end{array}$ & $0,931 * * * *$ & 0,248 & 0,000 & 0,307 & 0,061 & 0,092 \\
\hline $\begin{array}{l}\text { Le ménage habite dans le quartier } \\
\text { Magoanine « } \mathrm{B} »\end{array}$ & $0,597 * * * *$ & 0,213 & 0,005 & 0,214 & 0,067 & 0,092 \\
\hline $\begin{array}{l}\text { Le ménage habite dans le quartier } \\
\text { Magoanine « } \mathrm{C} »\end{array}$ & $0,337 * *$ & 0,194 & 0,082 & 0,127 & 0,069 & 0,109 \\
\hline $\begin{array}{l}\text { Le ménage habite dans le quartier } \\
\text { Albazine }\end{array}$ & 0,03 & 0,227 & 0,895 & 0,012 & 0,088 & 0,071 \\
\hline Constante & $-0,395$ & 0,338 & 0,242 & - & - & - \\
\hline $\begin{array}{l}\text { Nombre d'observations : } 871 \\
\text { Wald } \mathrm{Chi}^{2}(21): 208,89 \\
\text { Pseudo } \mathrm{R}^{2}: 29,58 \% \\
\text { AIC : } 1,020 \\
\text { Cas correctement prédits : } 75,55 \%\end{array}$ & $\mathrm{Pr}$ & $\begin{array}{l}\text { pseudol } \\
\mathrm{b}>\mathrm{Chi} 2 \\
\text { de Mac F } \\
\text { : }-490\end{array}$ & $\begin{array}{l}\text { elihood } \\
0,0000 \\
\text { iden : } 29 \\
267\end{array}$ & $\begin{array}{l}422,0 \\
\%\end{array}$ & & \\
\hline
\end{tabular}

$* * * *, * * *, * *, *$ significatifs respectivement à $1 \%, 5 \%, 10 \%$ et $15 \%$.

$d y / d x$ mesure les effets marginaux. X correspond aux valeurs moyennes des variables explicatives avec lesquelles ont été calculés les effets marginaux. Avec ces moyennes, la probabilité pour qu'un ménage soit raccordé est de 0,57678442 . Le fait que le chef de ménage soit analphabète diminue la probabilité d'être raccordé de 14,8 points de pourcentage comparativement à celui qui est alphabétisé. 
quand le chef de ménage dispose d'un niveau primaire, $65 \%$ avec un niveau secondaire et $90 \%$ avec un niveau supérieur.

Le fait d'être locataire influence négativement la probabilité que les ménages soient raccordés aux réseaux des POPs. En effet, les ménages non propriétaires de leur logement sont moins enclins à investir dans les frais de connexion. Par ailleurs, le raccordement à un réseau implique une démarche administrative qui nécessite des justificatifs que seul le propriétaire peut fournir. Enfin, certains propriétaires hésitent à supporter le coût de raccordement et donc à établir le contrat à leur nom puisqu'ils craignent le manque de solvabilité de leurs locataires pour le paiement des charges récurrentes (factures d'eau). Ainsi, bien souvent, à la question « Pourquoi n'êtes-vous pas raccordé à un réseau d'eau potable ? », les ménages locataires ou occupant à titre gratuit répondent :

« Je ne suis pas chez moi, alors je ne peux pas me raccorder. Je le ferai quand je serai chez moi. »

«Le propriétaire ne veux pas le faire.»

\section{Ou encore :}

« Ce n'est pas à moi de le faire, mais au propriétaire. Or il ne veut pas. »

Le niveau de richesse augmente la probabilité qu'un ménage soit raccordé à un réseau d'eau potable POP. Ce résultat va dans le sens attendu. L'indice de richesse apparaît être un élément décisif dans la décision de connexion des ménages d'autant que le coût d'installation du branchement est relativement élevé (en moyenne 1390 MTN, environ 35 euros). Au-delà des charges initiales, le ménage doit avoir la capacité à payer des factures mensuelles. À la question : " Pourquoi n'êtes-vous pas raccordé à un réseau d'eau potable? ", les réponses étaient majoritairement en lien avec l'insuffisance des moyens financiers :

« Je n'ai pas de moyens financiers. »

« Je suis pauvre et je ne peux pas payer le coût de raccordement initial. »

« Le POP qui est à côté de chez moi est trop cher. »

\section{Ou encore :}

« Je suis en train de me préparer, quand j'aurai réussi à rassembler le montant nécessaire pour me raccorder, je le ferai. »

Le calcul de l'effet marginal (c'est-à-dire le degré d'influence de la variable explicative sur la probabilité que le ménage soit raccordé à un réseau POP) révèle que, lorsque l'indice de richesse du ménage augmente d'une unité, la probabilité que ce dernier soit raccordé augmente de 0,066 point.

Le fait que le ménage ne dispose que d'un dollar par jour et par personne affecte négativement le fait qu'il soit raccordé à un réseau POP. Les réseaux développés par les opérateurs indépendants n'atteignent pas les plus pauvres. Cette variable, purement monétaire, considérée comme un critère d'extrême pauvreté, donne une approche complémentaire à celle fournie par l'indice de richesse basé sur les équipements du ménage (richesse matérielle). Sa significativité est 
intéressante en tant qu'instrument d'aide à la décision dans la lutte contre la pauvreté notamment dans le ciblage des subventions et des bénéficiaires des programmes. Dans les quartiers délaissés par l'opérateur officiel (AdeM), les bailleurs de fonds, qui subventionnent l'activité des POPs pour promouvoir le raccordement des plus pauvres, pourraient utiliser cet indicateur.

Le modèle démontre qu'une part élevée des branchés insatisfaits du service dans le quartier n'affecte pas négativement la décision de raccordement des ménages. Ceci laisse supposer que les insatisfactions aux autres sources étant plus importantes encore, le branchement privé reste la solution la plus souhaitable pour les ménages.

Les résultats révèlent des effets " quartiers ». Sur les treize dummy « quartier », dix sont significatives (huit agissant positivement et deux négativement). Concernant l'influence positive de certains quartiers dans le fait que les ménages soient raccordés, plusieurs arguments peuvent être avancés. D'abord, certains quartiers sont relativement mieux équipés en service d'électrification et, donc, plus investis par l'activité des POPs. C'est le cas des quartiers de Cumbeza (le plus riche de Marracuene), de Khongolote et de Magoanine « B ». Le fait d'habiter dans le quartier $1^{\text {er }}$ de Maio a également un impact positif dans la décision de raccordement, ce quartier étant constitué de chefs de ménage relativement jeunes (37 ans en moyenne). Les ménages des quartiers de Matèque, de Ndlavela et de Magoanine «A », dont la taille est relativement plus élevée (six personnes en moyenne), sont plus enclins à se brancher aux réseaux des POP. Magoanine « $\mathrm{C}$ » est un quartier qui, d'après l'analyse de la répartition de la richesse, est plus largement constitué de ménages « modestes » et « aisés » plus propices à être connectés. Seuls deux quartiers ont un impact négatif sur le raccordement des ménages : Guava (quartier qui connaît une forte part de branchés insatisfaits du service d'eau) et Matola Gare (95\% d'analphabètes).

La taille du ménage n'influence pas la décision de raccordement des ménages aux réseaux des POPs. Il s'agit pourtant, d'après la littérature, d'un facteur clé, dont l'influence peut varier dans les deux sens. Un nombre important de personnes peut désinciter un chef de ménage pauvre à choisir le branchement domiciliaire afin de rationner la consommation d'eau potable (la collecte aux sources alternatives permettant de mieux contrôler les quantités d'eau consommées). À l'inverse, plus le nombre de personnes dans le ménage est élevé, plus la quantité d'eau consommée augmente, ce qui incite le ménage à se raccorder pour faciliter la collecte de l'eau (temps, pénibilité et coût aux sources alternatives). Dans notre étude appliquée à Maputo, la taille du ménage varie entre quatre et six personnes, ce qui est relativement plus faible que dans les autres pays africains et explique probablement la non-influence de cette variable.

Afin de tester la robustesse du modèle aux hypothèses de revenus (indice de richesse, le fait de vivre avec moins d'un dollar par jour et par personne) ainsi qu'aux dummy « quartier », trois variantes ont été réalisées. La première variante supprime « le fait de vivre avec moins d'un dollar par jour et par personne » mais conserve $l^{\prime}$ « indice de richesse ${ }^{15} »$. Les variables, y compris les effets quartiers,

15. Une variante excluant l'indice de richesse et conservant la variable « vivre avec moins d'un dollar par jour et par personne » a été estimée mais le modèle ne présentait qu'un taux de prédiction de 69,46\% et un $\mathrm{R}^{2}$ de Mac Fadden de 18,7\%, ce qui n'est pas satisfaisant (les résultats étant disponibles auprès des auteurs). 
restent significatives et agissent dans le même sens ( $c f$. tableau A7, annexe). La seconde variante vise à tester la pertinence du recours à l'indice de richesse en remplaçant ce dernier par quatre nouvelles variables d'équipement ${ }^{16}$ : la variable d'opinion (part des branchés insatisfaits du service) perd sa significativité. En revanche, la taille du ménage devient significative ; plus elle augmente, plus les ménages sont enclins à se connecter (le branchement domiciliaire permettant de bénéficier de plus fortes quantités d'eau et d'une collecte facilitée). Toutes les autres variables (socioéconomiques et dummy " quartier ») influencent dans le même sens la probabilité que les ménages soient raccordés. Enfin, les quatre nouvelles variables d'équipement sont significatives et positives ( $c f$. tableau A9). Il apparaît donc pertinent de recourir à l'indice de richesse (matérielle) qui permet de capter de manière synthétique ces informations. La dernière variante est réalisée sans les variables dummy « quartiers ». À l'exception de la variable d'opinion qui perd sa significativité, les variables socioéconomiques restent significatives et influencent dans le même sens la probabilité que les ménages soient raccordés aux réseaux des POPs ( $c f$. tableau A10). Les taux de prédiction et les $\mathrm{R}^{2}$ de Mac Fadden des trois variantes étant élevés, le modèle est considéré comme robuste.

\section{CONCLUSION}

La situation de Maputo apparaît particulière au regard des autres pays d'Afrique subsaharienne, notamment du fait de la présence des POPs dans les zones non desservies par l'opérateur officiel.

Les indicateurs de revenu (richesse matérielle et extrême pauvreté) sont des facteurs clés qui influencent la décision de raccordement des ménages aux réseaux des POPs. La probabilité que le ménage se raccorde augmente avec l'indice de richesse et diminue lorsque le revenu par tête est inférieur à un dollar. Les réseaux d'eau potable ne sont donc toujours pas accessibles aux ménages les plus pauvres malgré les programmes de subvention. Par ailleurs, le fait que le chef de ménage soit analphabète influence négativement le choix de raccordement. $\mathrm{Ce}$ résultat signifie que la réussite des programmes de promotion des branchements privés est conditionnée par la mise en place de campagnes de sensibilisation de la population à l'intérêt sanitaire d'un accès amélioré à l'eau potable. Lorsque les ménages ne sont que locataires de leur logement, ceux-ci sont moins enclins à se connecter aux réseaux des POPs. Pourtant, le statut de locataire est très courant à Maputo, ce qui est un réel frein. De nouvelles conditions d'accès pourraient être imaginées pour faciliter et inciter les ménages non propriétaires de leur logement à choisir cette option. L'analyse des effets quartiers révèle que ces derniers ne sont pas homogènes tant du point de vue de la répartition des quartiles de richesse que du point de vue de l'accès aux réseaux d'eau potable. Par ailleurs, une réflexion conjointe sur la stratégie d'électrification et d'extension

16. Une variable binaire prenant la valeur 1 si le ménage dispose d'un congélateur sinon 0 , une variable binaire prenant la valeur 1 si le ménage dispose d'un micro-onde sinon 0 , une variable prenant la valeur 1 si le ménage dispose d'un ventilateur sinon 0 , une variable binaire prenant la valeur 1 si le ménage dispose d'un réfrigérateur sinon 0 . Un test de corrélation a été effectué (cf. tableau A8, annexe). 
des réseaux d'eau potable devrait être menée par les autorités et les bailleurs de fonds pourvoyeurs de subventions pro-pauvres. La significativité du revenu par tête inférieur à un dollar (mesure de l'extrême pauvreté monétaire des ménages) montre qu'il s'agit d'un critère pertinent pour le ciblage des bénéficiaires des programmes sociaux même si l'approche matérielle de la richesse reste le meilleur proxy du revenu. Ce résultat n'avait pas été démontré dans la littérature relative à l'analyse de la demande en eau des ménages.

Notre étude porte sur des quartiers délaissés par l'opérateur officiel. Mais aujourd'hui, dans de nombreux quartiers périphériques de Maputo, l'AdeM et le FIPAG développent également un réseau en parallèle des POPs. Cette concurrence devrait être régulée si les autorités décidaient d'intégrer officiellement les POPs dans le marché de distribution d'eau potable. Whittington [2003] a déjà suggéré l'idée selon laquelle les gouvernements devraient délivrer des autorisations légales aux opérateurs indépendants avec des zones d'intervention bien délimitées. Estache et Kouassi [2002] ont également montré que les capacités institutionnelles des PED (à travers la mise en place d'une «bonne » gouvernance) représentaient un élément clé de la performance des firmes hydrauliques. D'autres études comme celle développée par Sansom [2006] ont souligné l'importance du rôle des gouvernements en matière de régulation du secteur de l'eau lorsque différents acteurs coexistent (opérateur officiel, opérateurs privés et société civile). Au-delà des questions relatives au partage du marché et à l'organisation des partenariats public-privé (Davis [2005]), demeure la question de la régulation des tarifs (Nickson et Vargas [2002] ; Whittington, Boland et Foster [2003]) et de celle de la qualité (de service et de la ressource). En effet, le coût d'accès ainsi que le prix de l'eau fournie par les réseaux (AdeM ou POPs) devraient être les mêmes dans tous les quartiers. La qualité du service (continuité) mais également celle de la ressource devraient être également garanties pour tous selon que le fournisseur soit l'opérateur officiel ou les POPs, d'autant que la légitimité de ces derniers dépend fortement de leur capacité à délivrer un service de qualité à un coût équitable. Cet aspect a déjà fait débat dans la littérature. Si certains auteurs valorisent le rôle joué par les opérateurs indépendants dans l'amélioration de l'accès (Njiru et Albu [2004]), d'autres comme Jaglin [2002] ont souligné certaines limites aux initiatives privées de types participatif et communautaire. En effet, bien qu'ils permettent d'améliorer ponctuellement l'accès à l'eau potable des plus démunis dans des quartiers délaissés, ils ne permettent pas toujours de mobiliser les capitaux nécessaires au développement des réseaux. Par ailleurs, en l'absence de régulation, ces systèmes risquent d'institutionnaliser l'existence de deux types de service dont celui destiné aux plus démunis reste contraignant (Baker et Trémolet [2003] et Solo [1999]). C'est précisément ce risque qui justifie l'intérêt croissant que portent les bailleurs de fonds aux actions des POPs en leur octroyant des subventions (Kariuki et Schwart [2005]), le ciblage des bénéficiaires restant l'enjeu essentiel.

\section{RÉFÉRENCES BIBLIOGRAPHIQUES}

AhMAD J.K., BishwANATH G., MisRa S. [2005], « Value of arsenic-free drinking water to rural households in Bangladesh », Journal of Environmental Management, 74, p. 173-185. 
Altaf M.A, Whittington D., Jamal H., Smith K. [1993], « Rethinking Rural Water Supply Policy in Punjab, Pakistan », Water Resource Research, 29, p. 1943-1954.

BAKER B., TREMOLET S. [2003], "Regulation of the quality of infrastructure services in developing countries », dans BROOK P.J. et IRWIN T.C. (eds), Infrastructure for Poor People: Public Policy for Private Provision, The World Bank, Washington D.C., p. 233-275.

BASANI M., Isham J., ReILly B. [2008], « The Determinants of Water Connection and Water Consumption: Empirical Evidence from a Cambodian Household Survey ", World Development, 36 (5), p. 953-968.

Blanc A., CAVE J., ChaponNIERE E. [2009], « Les petits opérateurs privés de la distribution d'eau à Maputo : d'un problème à une solution ? Regards croisés », Document de travail AFD, 85, Paris.

Briand A., NaUges C., Strand J., TRavers M. [2010], « The Impact of Tap Connection on Water Consumption Patterns: The Case of Household Water Use in Dakar, Senegal », Environment and Development Economic, 15 (1), p. 107-126.

Briand A., NaUges C., Travers M. [2009], « Choix d'approvisionnement en eau des ménages de Dakar : une étude économétrique à partir de données d'enquête », Revue d'Économie du Développement, 3, p. 83-108.

Briscoe J., De CASTRO P.F., GrifFin C., NORTH J., Olsen O. [1990], « Toward Equitable and Sustainable Rural Water Supplies: A Contingent Valuation Study in Brazil », The World Bank Economic Review, 4 (2), p. 115-143.

Brocklehurst C., JANSSENS J.G. [2004], « Innovative Contracts, Sound Relationships: Urban Water Sector in Senegal », WSSB Discussion Paper Series, Paper $n^{\circ} 1$.

BudDS J., MC GRANAHAN G. [2003], « Are the debates on water privatization missing the point? Experiences from Africa, Asia and Latin America », Environment and Urbanization, 15 (2), p. 87-113.

CAHUZAC E., BONTEMPS C. [2008], « Stata par la pratique : statistiques, graphiques et éléments de programmation $»$, Stata Press.

CAirncross S., ClifF J.L. [1987], «Water Use and Health in Mueda, Mozambique », Transactions of the Royal Society of Tropical Medicine and Hygiene, 81, p. 51-54.

Clarke G. R. G., Kosec K., Wallsten S. [2004], Has Private Participation in Water and Sanitation Improved Coverage? Empirical Evidence from Latin America, AEI Brookings Joint Center for Regulatory Studies.

Collignon B., [1998], « The Potential and the Limits of Private Water Providers Independent Markets in Francophone Africa », UNDP/World Bank Water and Sanitation Program, Washington D.C.

Collignon B., Vezina M. [2000], « Independent Water and Sanitation Providers in African Cities: Full Report of a Ten Country Study », Water and Sanitation Program, World Bank, Washington D.C.

CONAN H. [2003], « Scope and Scale of Small Scale Independent Private Water Providers in 8 Asian Cities », Asian Development Bank, Manille.

Conan H., Paniagua M. [2003], « The Role of Small Scale Private Water Providers in Serving the Poor », Asian Development Bank, Manille.

CURTIS F. [1986], « Women and the Transport of Water », Londres, Intermediate Technology Publications.

DardenNe B., Razafinjato G., Dardenne L. [2008], " Le modèle technique et commercial des petits opérateurs de réseaux privés d'eau à Maputo », Document de travail AFD, Paris.

DAVIS, J. [2005], " Private Sector Participation in the Water and Sanitation Sector », Annual Review of Environment and Resources, 30, p. 145-183.

ESTACHE A., KoUASSI E. [2002], « Sector Organization, Governance, and Inefficiency of African Water Utilities », World Bank Policy Research Working Paper, 2890.

Filmer D., PritchetT L.H. [2001], « Estimating Wealth Effects without Expenditure Data or Tears: An Application to Educational Enrolments in States of India », Demography, 38 (1), p. 115-132. 
Franceys R., Weitz A. [2003], « Public-private community partnerships in infrastructure for the poor », Journal of International Development, 15 (8), p. 1083-1098.

FRANCEYS R. [1997], « Private Waters - a Bias towards the Poor», DIFD/WEDC/IHE.

GINISTRY K. [2009], « Les petits opérateurs privés d'approvisionnement en eau à Maputo: inscription spatiale et acteurs du territoire », Document de travail AFD, Paris.

HART K. [1970], "Small-scale entrepreneurs in Ghana and development planning », Journal of Development Studies, 6 (4), p. 104-120.

JAGLIN S. [2002], « The Right to water versus cost recovery: participation, urban water supply and the poor in sub-Saharan Africa », Environment and Urbanization, 14 (1), p. 231-245.

KARIUKI, M., SchWARTZ J. [2005], « Small-Scale Private Service Providers of Water Supply and Electricity: a review of Incidence, Structure, Pricing, and Operating Characteristics ", World Bank Policy Research Working Paper, 3727.

LARSON B., MinTEN B., RAZAFINDRALAMBO R. [2006], « Unravelling the Linkages between the Millennium Development Goals for Poverty, Education, Access to Water and Household Water Use in Developing Countries: Evidence from Madagascar », Journal of Development Studies, 42 (1), p. 22-40.

MADANAT S., HUMPLICK F. [1993], « A model of household choice of water demand supply systems in developing countries », Water Resources Research, 29, p. 1353-1358.

McGranahan G., Nitru C., Albu M., Smith M., Mitlin, D. [2006], « How small water enterprises (SWEs) can contribute to the Millennium Development Goals: Evidence from Accra, Dar Es Salaam, Khartoum and Nairobi », WEDC Loughborough University, Loughborough (UK).

McGranahan G., Owen L. D. [2006], « Getting Local Water and Sanitation Companies to Improve Water and Sanitation Provision for the Urban Poor », Discussion Paper: Water-4, International Institute for Environment and Development, Londres.

Mu X., WhitTington D., Briscoe J., BARRON W. [1990], « Estimating the Willingness to Pay for Water Services in Developing Countries: A Case Study of the Use of Contingent Valuation Surveys in Southern Haiti », Economic Development and Cultural Change, 38 (2), p. 293-311.

Mu X., Whittington D., Roche R. [1990], «Calculating the Value of Time Spent Collecting Water: Some Estimates for Uganda, Kenya », World Development, 18 (2), p. 226-280.

Nauges C., Whittington, D. [2010], « Estimation of Water Demand in Developing Countries: An Overview », The World Bank Research Observer, 25 (2), p. 263-294.

NAUges C., STRAND J. [2007], « Estimation of Non-Tap Water Demand in Central American cities », Resource and Energy Economics, 29 (3), p. 165-182.

NAuges C., VAn Den Berg C. [2009], « Demand for Piped and Non-Piped Water Supply Services: Evidence from Southwest Sri Lanka », Environmental and Resource Economics, (42) 4, Springer Netherlands, p. 535-549.

NICKSON A., VARGAS C. [2002], «The limitations of Water Regulation: the failure of the Cochabamba Concession in Bolivia », Bulletin of Latin American Research, 21 (1), p. 99-120.

NJIRU, C., ALBU, M. [2004], « Improving access to water through support to small water providing enterprises », Small Enterprise Development, 15 (2), p. 30-36.

SANSOM, K. [2006], « Government engagement with non-state providers of water and sanitation services », Public Administration and Development, 26, p. 207-217.

SNELL S. [1998], « Small-Scale entrepreneurs in water and sanitation: profiles and typologies », WSP Working Paper, UNDP/Water and Sanitation Program.

Solo T.M. [2003], « Independent Water Entrepreneurs in Latin America: the Other Private Sector in Water Services », World Bank, Washington D.C.

Solo T.M. [1999], "Small-Scale Entrepreneurs in the Urban Water and Sanitation Market », Environment and Urbanization, 11 (1), p. 117-132.

WhitTington D. [2003], « Municipal Water Pricing and Tariff Design: a reform agenda for South Asia », Water Policy, 5, p. 61-76. 
Whittington D., Boland J., Foster V. [2003], « Water tariffs and subsidies in South Asia: understanding the basic ", Public Private Infrastructure Advisory Facility (PPIAF) and the Water and Sanitation Program (WSP), The World Bank, Washington D.C.

WhitTington D., LAURIA D.T., MU X. [1991], «A study of Water Vending and Willingness to Pay for Water in Onitsha, Nigeria », World Development, 19 (2/3), p. 179-198.

ANNEXE

Tableau A1. Les variables recodées pour la construction de l'indice de richesse

\begin{tabular}{|c|c|}
\hline Variables recodifiées & Signification (1 si oui, 0 si non) \\
\hline eau_couran d & le ménage possède-t-il un bain douche interne avec eau courante ? \\
\hline wc_recod & le ménage possède-t-il un WC moderne? \\
\hline drenage_eu d & le ménage possède-t-il une fosse septique? \\
\hline branc_elec $\sim \mathrm{d}$ & le ménage possède-t-il un branchement électrique? \\
\hline four_recod & le ménage possède-t-il un four? \\
\hline aspirateur $\sim \mathrm{d}$ & le ménage possède-t-il un aspirateur? \\
\hline lave_vaiss $\sim$ d & le ménage possède-t-il un lave-vaisselle? \\
\hline fer_elect_ d & le ménage possède-t-il un fer à repasser électrique? \\
\hline lave_linge $\sim \mathrm{d}$ & le ménage possède-t-il un lave-linge ? \\
\hline vido_recod & le ménage possède-t-il un lecteur DVD ? \\
\hline ordinateur $\sim \mathrm{d}$ & le ménage possède-t-il un ordinateur? \\
\hline micro_onde $\sim \mathrm{d}$ & le ménage possède-t-il un micro-onde? \\
\hline ventilateu $\sim$ d & le ménage possède-t-il un ventilateur? \\
\hline climatiseu d & le ménage possède-t-il un climatiseur? \\
\hline congelateu $\sim \mathrm{d}$ & le ménage possède-t-il un congélateur? \\
\hline mach_cou_r $\sim d$ & le ménage possède-t-il une machine à coudre ? \\
\hline tlph_fixe_ d & le ménage possède-t-il un téléphone fixe? \\
\hline tl_mobile_ d & le ménage possède-t-il un téléphone mobile? \\
\hline radio_recod & le ménage possède-t-il une radio ? \\
\hline tlvision_r $\sim \mathrm{d}$ & le ménage possède-t-il une télévision? \\
\hline rfrigrateu $\sim \mathrm{d}$ & le ménage possède-t-il un réfrigérateur? \\
\hline bicyclette $\sim$ d & le ménage possède-t-il une bicyclette? \\
\hline motocyclet $\sim \mathrm{d}$ & le ménage possède-t-il une motocyclette? \\
\hline voiture_re $\sim d$ & le ménage possède-t-il une voiture? \\
\hline habitat_1_ d & le ménage possède-t-il une maison moderne? \\
\hline habitat_2_ d & le ménage possède-t-il un habitat précaire ? \\
\hline murs_cimen $\sim \mathrm{d}$ & le ménage possède-t-il un mur en ciment? \\
\hline murs_palme $\sim d$ & le ménage possède-t-il un mur en palmette? \\
\hline toit_zinc_ $\sim \mathrm{d}$ & le ménage possède-t-il un toit en zinc ? \\
\hline toit_cimen $\sim$ d & le ménage possède-t-il un toit en ciment? \\
\hline toit_palme $\sim \mathrm{d}$ & le ménage possède-t-il un toit en palmette? \\
\hline sol_ciment $\sim$ d & le ménage possède-t-il un sol en ciment? \\
\hline sol_terre_ $\sim \mathrm{d}$ & le ménage possède-t-il un sol en terre? \\
\hline
\end{tabular}


Tableau A2. Méthode de calcul de l'indice de richesse

\begin{abstract}
$\mathrm{S} i j=[(\text { valeur de } j \text { pour l'individu } i-\text { moyenne de } j) / \text { écart type } j]^{*}$ score factoriel $j$.
$\mathrm{S} i j=$ score du ménage $i$ pour le bien $j$.

Vij est la valeur du bien $j$ pour l'individu $i$. Elle est égale à 0 si l'individu ne possède pas le bien et 1 s'il possède le bien.

La moyenne du bien $j$ et son écart type sont calculés sur l'échantillon total. L'objectif est de déterminer pour chaque ménage un score global (somme de tous les scores) qui correspond à son indice de richesse.

IR $i=\sum$ Score de tous les biens, IR $i$ : score ou poids du ménage $i$.
\end{abstract}

Source : Morel A., L’Huissier A. [2003], Rapport final : « Gestion domestique des eaux usées et des excrétas : étude des pratiques et comportements, des fonctions de demande, de leur mesure en situation contingente et de leur opérationnalisation », PDM, PS-eau.

Tableau A3. Les données du calcul des scores

\begin{tabular}{|c|c|c|c|c|c|}
\hline \multirow[b]{2}{*}{ Variables } & \multirow[b]{2}{*}{ Moyenne } & \multirow[b]{2}{*}{ Écart type } & \multirow[b]{2}{*}{ Score factoriel } & \multicolumn{2}{|c|}{ Score du ménage $i$} \\
\hline & & & & $\begin{array}{l}\text { S'il possède } \\
\text { le bien }\end{array}$ & $\begin{array}{l}\text { S'il ne possède } \\
\text { pas le bien }\end{array}$ \\
\hline drenage_eu d & 0,5113636 & 0,5001551 & 0,4201 & $-0,429514461$ & 0,410424989 \\
\hline branc_elec $\sim \mathrm{d}$ & 0,6575654 & 0,4747943 & 0,645 & $-0,893291438$ & 0,465191593 \\
\hline four_recod & 0,3215909 & 0,4673525 & 0,5717 & $-0,393393675$ & 0,829879978 \\
\hline aspirateur $\sim \mathrm{d}$ & 0,0079545 & 0,0888833 & 0,1767 & $-0,015813546$ & 1,97218645 \\
\hline lave_vaiss $\sim \mathrm{d}$ & 0,1090909 & 0,3119305 & 0,5637 & $-0,1971418$ & 1,609991519 \\
\hline lave_linge $\sim \mathrm{d}$ & 0,0159272 & 0,1252652 & 0,2811 & $-0,035741259$ & 2,208297788 \\
\hline vido_recod & 0,3465909 & 0,4761547 & 0,5701 & $-0,414973268$ & 0,782326685 \\
\hline ordinateur $\sim \mathrm{d}$ & 0,1002278 & 0,3004746 & 0,5228 & $-0,174387765$ & 1,565526358 \\
\hline micro_onde $\sim$ d & 0,0613636 & 0,2401326 & 0,491 & $-0,125470376$ & 1,919233259 \\
\hline ventilateu $\sim \mathrm{d}$ & 0,303551 & 0,4600546 & 0,5688 & $-0,375302864$ & 0,861072123 \\
\hline climatiseu $\sim \mathrm{d}$ & 0,0342075 & 0,1818656 & 0,3538 & $-0,066547019$ & 1,878845623 \\
\hline congelateu $\sim \mathrm{d}$ & 0,31813 & 0,466016 & 0,5592 & $-0,381742893$ & 0,818215907 \\
\hline mach_cou_r $\sim d$ & 0,0445205 & 0,2063664 & 0,1354 & $-0,029210548$ & 0,626904013 \\
\hline tlph_fixe_ $\sim d$ & 0,0307167 & 0,1726474 & 0,2138 & $-0,038038398$ & 1,200323721 \\
\hline tl_mobile_ $\sim d$ & 0,8892694 & 0,3139775 & 0,3151 & $-0,892448624$ & 0,111126473 \\
\hline radio_recod & 0,6556442 & 0,4754289 & 0,4803 & $-0,662361731$ & 0,347883965 \\
\hline rfrigrateu $\sim \mathrm{d}$ & 0,238806 & 0,4265989 & 0,5773 & $-0,32316704$ & 1,030094771 \\
\hline bicyclette $\sim$ d & 0,0798176 & 0,2711652 & 0,3331 & $-0,098048137$ & 1,130354328 \\
\hline motocyclet $\sim \mathrm{d}$ & 0,0207852 & 0,1427471 & 0,1927 & $-0,02805877$ & 1,321881089 \\
\hline voiture_re $\sim \mathrm{d}$ & 0,1264237 & 0,3325157 & 0,5595 & $-0,212723971$ & 1,469903345 \\
\hline murs_palme $\sim \mathrm{d}$ & 0,1045455 & 0,3061408 & $-0,316$ & 0,107912366 & $-0,924292424$ \\
\hline toit_cimen $\sim \mathrm{d}$ & 0,0477273 & 0,2133098 & 0,2815 & $-0,062984612$ & 1,256692215 \\
\hline toit_palme $\sim \mathrm{d}$ & 0,0011364 & 0,03371 & $-0,0122$ & 0,000411275 & $-0,361499137$ \\
\hline sol_terre_ d & 0,0602273 & 0,2380427 & $-0,2272$ & 0,057483983 & $-0,896966626$ \\
\hline
\end{tabular}


Graphique A1. Répartition des quartiles de l'indice de richesse dans les différents quartiers enquêtés de Maputo (en \%)

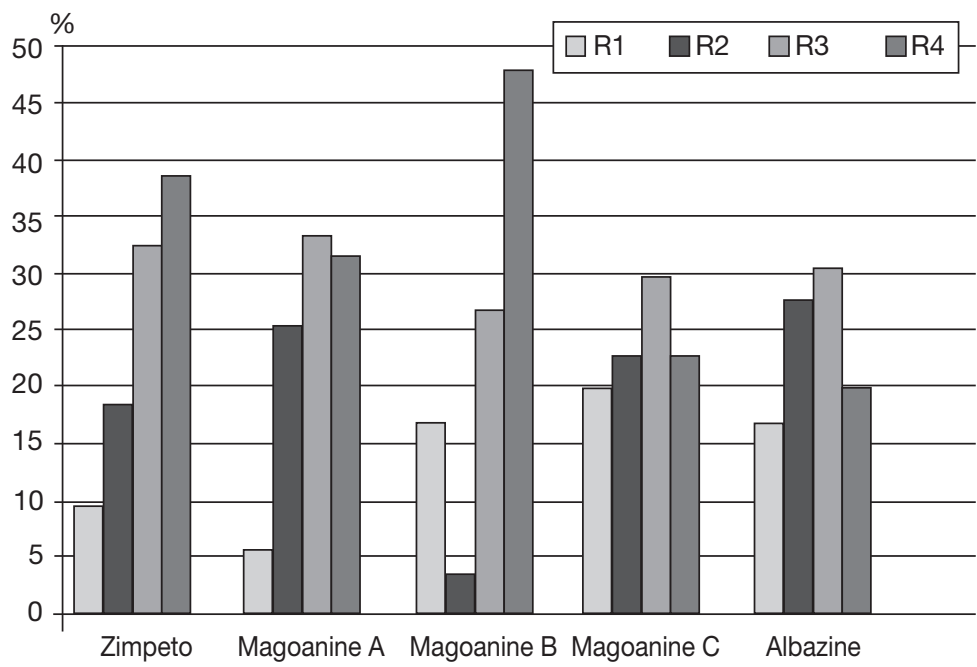

Graphique A2. Répartition des quartiles de l'indice de richesse dans les différents quartiers enquêtés de Matola (en \%)

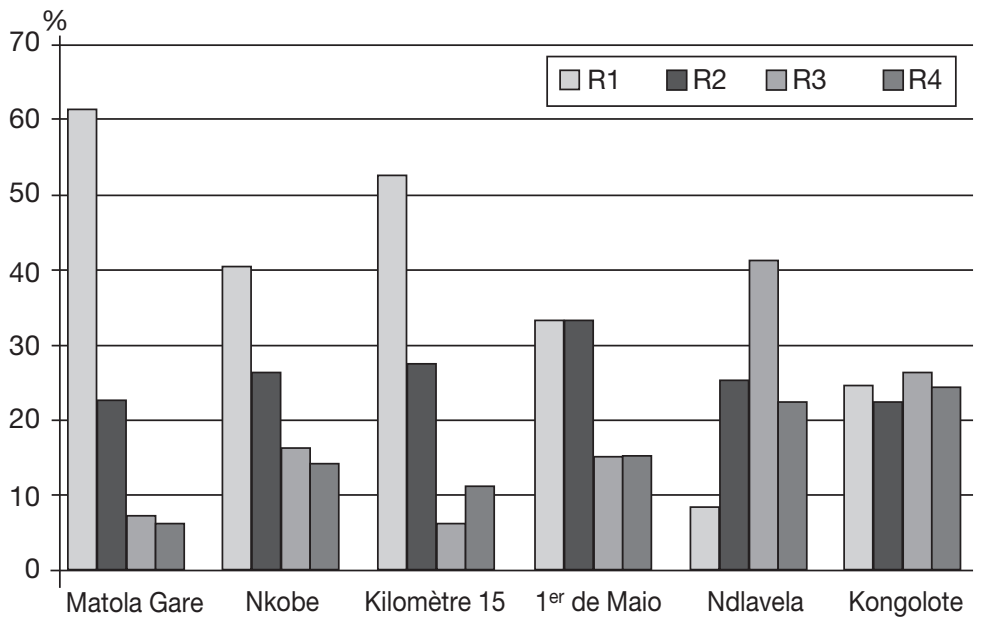


Graphique A3. Pourcentage de ménages ayant accès à l'électricité et au branchement privé selon les quartiers enquêtés

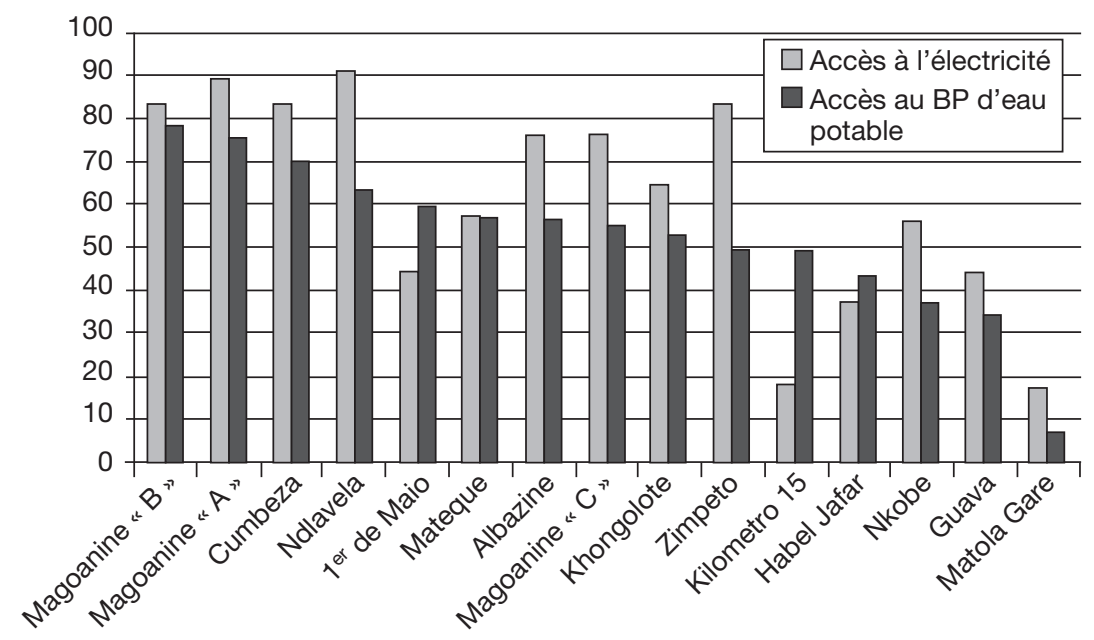

Graphique A4. Répartition des quartiles de l'indice de richesse dans les différents quartiers enquêtés de Marracuene (en \%)

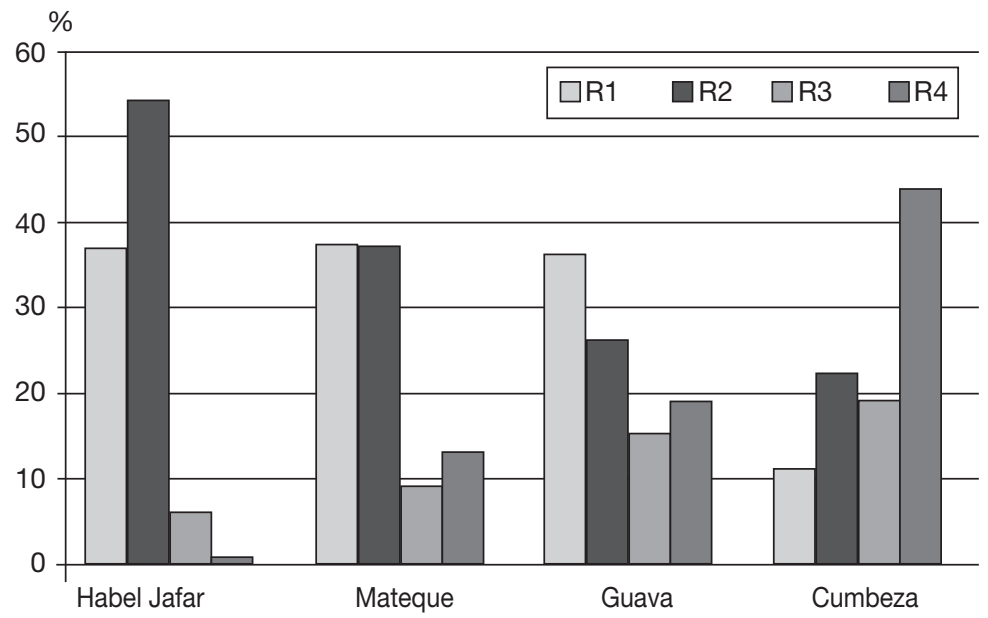


Tableau A4. Test de multicolinéarité (VIF)

\begin{tabular}{|c|c|c|}
\hline Variables & VIF & $1 / \mathrm{VIF}$ \\
\hline La part des branchés insatisfaits du service dans le quartier & 5,59 & 0,178791 \\
\hline Le ménage habite dans le quartier Matola Gare & 2,38 & 0,420492 \\
\hline Le ménage habite dans le quartier Khongolote & 2,28 & 0,438145 \\
\hline Le ménage habite dans le quartier Ndlavela & 1,93 & 0,517206 \\
\hline Le ménage habite dans le quartier Cumbeza & 1,86 & 0,538803 \\
\hline Le ménage habite dans le quartier Magoanine « $\mathrm{A}$ » & 1,85 & 0,539346 \\
\hline Le ménage habite dans le quartier Guava & 1,78 & 0,560839 \\
\hline Le ménage habite dans le quartier Kilomètre 15 & 1,77 & 0,565828 \\
\hline Le ménage habite dans le quartier Albazine & 1,69 & 0,59268 \\
\hline Le ménage habite dans le quartier Magoanine « $\mathrm{C} »$ & 1,65 & 0,606877 \\
\hline Le ménage habite dans le quartier Magoanine « B » & 1,62 & 0,616797 \\
\hline L'indice de richesse du ménage & 1,56 & 0,639491 \\
\hline Le ménage habite dans le quartier $1^{\mathrm{er}}$ de Maio & 1,51 & 0,661685 \\
\hline Le ménage habite dans le quartier Matèque & 1,49 & 0,669996 \\
\hline $\begin{array}{l}\text { Le ménage vit avec moins d'un dollar par jour } \\
\text { et par personne }\end{array}$ & 1,44 & 0,696372 \\
\hline La taille du ménage & 1,32 & 0,755861 \\
\hline Le ménage habite dans le quartier Nkobe & 1,32 & 0,756918 \\
\hline Le chef de ménage est analphabète $(1 / 0)$ & 1,23 & 0,8101 \\
\hline L'âge du chef de ménage & 1,23 & 0,811366 \\
\hline Le chef de ménage est une femme $(1 / 0)$ & 1,15 & 0,871451 \\
\hline Le ménage est locataire de son logement $(1 / 0)$ & 1,06 & 0,941154 \\
\hline Mean VIF & 1,8 & \\
\hline
\end{tabular}

Il y a multicolinéarité dans la régression lorsque la VIF la plus élevée est supérieure à 10, ce qui n'est pas le cas. On conclut à une absence de multicolinéarité (Cahuzac E. et Bontemps C. [2008]). 
Tableau A5. Test d'endogénéité

L'indice de richesse du ménage = le ménage possède un congélateur + le ménage possède un micro-onde + le ménage possède un ventilateur + le ménage possède un réfrigérateur

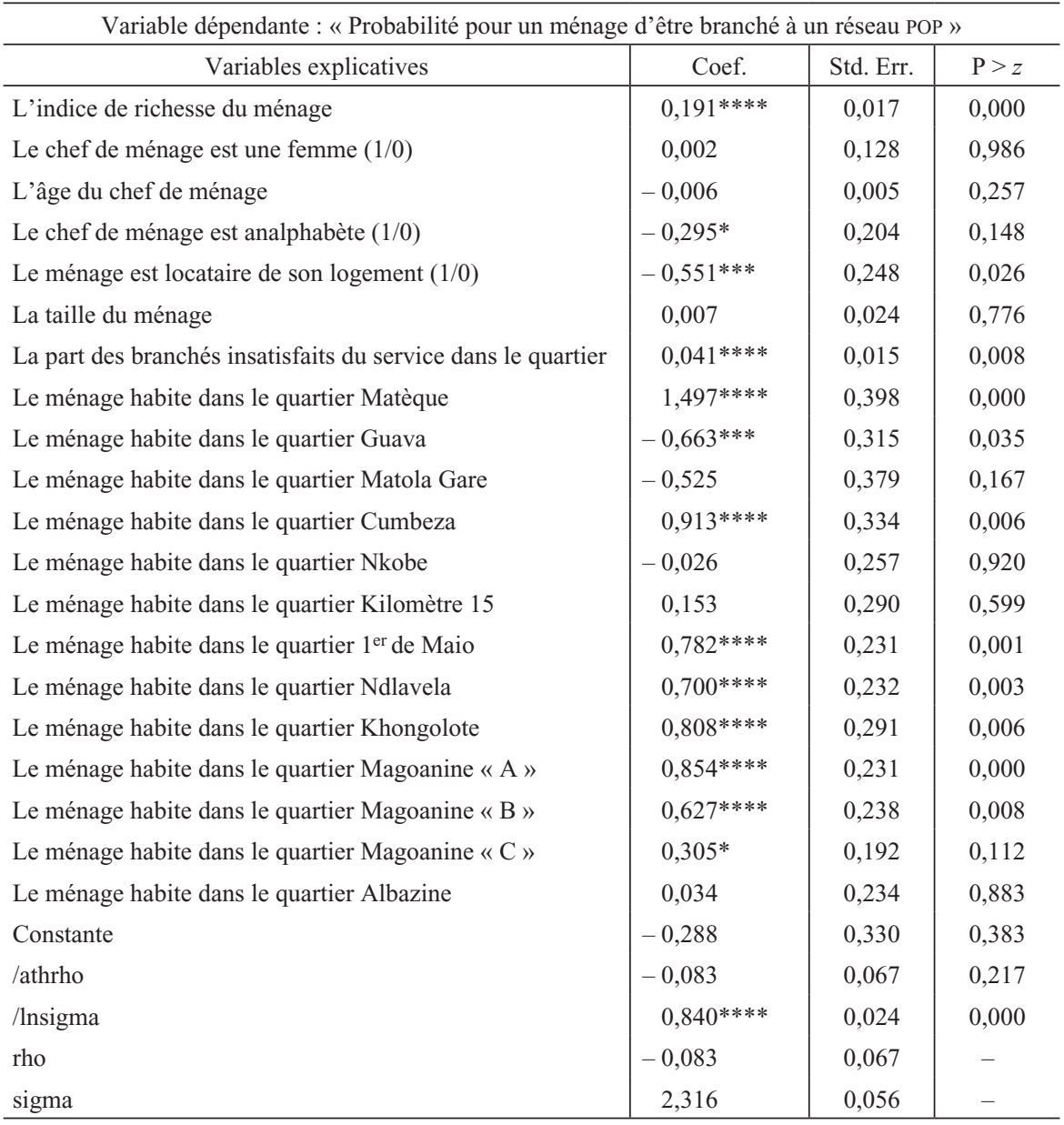

Nombre d'observations : 852

Log pseudolikelihood : $-2341,3591$

$\operatorname{LRChi}^{2}(20): 215,87$

Prob $>\mathrm{Chi}^{2}: 0,0000$

Test Wald (exogénéité) (/athrho $=0): \mathrm{Chi}^{2}(1)=1,52 \mathrm{Prob}>\mathrm{Chi}^{2}=0,2173$

Variable à instrumentaliser : l'indice de richesse du ménage.

Variables instrumentales : le ménage possède un congélateur + le ménage possède un micro-onde + le ménage possède un ventilateur + le ménage possède un réfrigérateur.

Prob $>\mathrm{Chi}^{2}=0,2173$, ce qui est supérieur 0,05 , alors on peut rejeter l'hypothèse H0, c'est-à-dire l'hypothèse que l'indice de richesse est endogène. 
Tableau A6. Statistiques descriptives des variables explicatives selon les quartiers enquêtés

\begin{tabular}{|c|c|c|c|c|c|c|c|c|c|c|c|c|}
\hline \multirow[b]{2}{*}{ Quartier } & \multicolumn{2}{|c|}{ Femme } & \multirow{2}{*}{\begin{tabular}{|c|} 
Âge \\
Moyenne \\
\end{tabular}} & \multicolumn{2}{|c|}{$\begin{array}{c}\mathrm{CM} \\
\text { analphabète }\end{array}$} & \multicolumn{2}{|c|}{$\begin{array}{l}\text { Ménage } \\
\text { locataire }\end{array}$} & \multirow{2}{*}{$\begin{array}{c}\text { Taille } \\
\text { du } \\
\text { ménage }\end{array}$} & \multirow{2}{*}{$\begin{array}{c}\begin{array}{c}\text { Indice } \\
\text { de } \\
\text { richesse }\end{array} \\
\text { Moyenne }\end{array}$} & \multicolumn{2}{|c|}{$\begin{array}{l}\text { Moins } \\
\text { d'un } \\
\text { dollar } \\
\text { par jour } \\
\text { et par } \\
\text { personne }\end{array}$} & \multirow[t]{2}{*}{$\begin{array}{c}\text { Part des } \\
\text { raccordés } \\
\text { insatis- } \\
\text { faits du } \\
\text { service } \\
\text { d'eau } \\
\text { Moyenne }\end{array}$} \\
\hline & Oui & Non & & Oui & Non & Oui & Non & & & Oui & Non & \\
\hline Habel Jafar & 80 & 20 & 39 & 85 & 15 & 97 & 3 & 4 & $-3,2$ & 32 & 68 & 23 \\
\hline Matèque & 67 & 33 & 51 & 90 & 10 & 100 & 0 & 6 & $-2,1$ & 52 & 48 & 0 \\
\hline Guava & 71 & 29 & 41 & 90 & 10 & 88 & 12 & 5 & $-0,6$ & 51 & 49 & 21 \\
\hline $\begin{array}{l}\text { Matola } \\
\text { Gare }\end{array}$ & 88 & 12 & 41 & 95 & 5 & 92 & 8 & 5 & 2,2 & 58 & 42 & 0 \\
\hline Cumbeza & 77 & 23 & 38 & 85 & 15 & 97 & 3 & 5 & $-3,5$ & 43 & 57 & 0 \\
\hline Nkobe & 71 & 29 & 37 & 88 & 12 & 98 & 2 & 5 & $-1,8$ & 63 & 37 & 13 \\
\hline $\mathrm{Km} 15$ & 63 & 37 & 38 & 85 & 15 & 95 & 5 & 5 & $-2,8$ & 62 & 38 & 21 \\
\hline $1^{\mathrm{er}}$ de Maio & 79 & 21 & 37 & 93 & 7 & 95 & 5 & 5 & $-1,3$ & 49 & 51 & 8 \\
\hline Ndlavela & 76 & 24 & 41 & 92 & 8 & 92 & 8 & 6 & 0,7 & 53 & 47 & 4 \\
\hline $\begin{array}{l}\text { Khongo- } \\
\text { lote }\end{array}$ & 81 & 19 & 39 & 88 & 12 & 95 & 5 & 5 & 0,3 & 56 & 44 & 0 \\
\hline Zimpeto & 78 & 22 & 45 & 91 & 9 & 95 & 5 & 6 & 1,7 & 58 & 42 & 5 \\
\hline $\begin{array}{l}\text { Magoanine } \\
\text { «A » }\end{array}$ & 81 & 19 & 41 & 91 & 9 & 99 & 1 & 6 & 1,6 & 54 & 46 & 5 \\
\hline $\begin{array}{l}\text { Magoanine } \\
« B »\end{array}$ & 78 & 22 & 42 & 96 & 4 & 94 & 6 & 5 & 3,0 & 69 & 31 & 13 \\
\hline $\begin{array}{l}\text { Magoanine } \\
« \mathrm{C} »\end{array}$ & 80 & 20 & 39 & 95 & 5 & 99 & 1 & 5 & $-0,3$ & 55 & 45 & 10 \\
\hline Albazine & 84 & 16 & 39 & 97 & 3 & 95 & 5 & 5 & 0,2 & 56 & 44 & 17 \\
\hline
\end{tabular}


Tableau A7. Estimation avec seulement l'indice de richesse

(«le ménage vit avec moins d'un dollar par jour et par personne » exclu) : variante 1

\begin{tabular}{l|c|c|c}
\hline \multicolumn{4}{c}{ Variable dépendante : « Probabilité pour un ménage d'être branché à un réseau POP » } \\
\hline \multicolumn{1}{c}{ Variables explicatives } & Coef. & Std. Err. & $\mathrm{P}>z$ \\
\hline Le chef de ménage est une femme (1/0) & 0,024 & 0,131 & 0,853 \\
L'âge du chef de ménage & $-0,004$ & 0,005 & 0,364 \\
Le chef de ménage est analphabète (1/0) & $-0,348^{* * *}$ & 0,202 & 0,086 \\
Le ménage est locataire de son logement (1/0) & $-0,564^{* * *}$ & 0,272 & 0,038 \\
La taille du ménage & 0,004 & 0,024 & 0,868 \\
L'indice de richesse du ménage & $0,184^{* * * *}$ & 0,017 & 0,000 \\
La part des branchés insatisfaits du service dans le quartier & $0,039^{* * *}$ & 0,015 & 0,011 \\
Le ménage habite dans le quartier Matèque & $1,605^{* * * *}$ & 0,389 & 0,000 \\
Le ménage habite dans le quartier Guava & $-0,604^{* * *}$ & 0,304 & 0,047 \\
Le ménage habite dans le quartier Matola Gare & $-0,596^{* *}$ & 0,355 & 0,093 \\
Le ménage habite dans le quartier Cumbeza & $0,904^{* * * *}$ & 0,307 & 0,003 \\
Le ménage habite dans le quartier Nkobe & $-0,028$ & 0,258 & 0,914 \\
Le ménage habite dans le quartier Kilomètre 15 & 0,216 & 0,283 & 0,444 \\
Le ménage habite dans le quartier 1er de Maio & $0,764^{* * * *}$ & 0,237 & 0,001 \\
Le ménage habite dans le quartier Ndlavela & $0,701^{* * * *}$ & 0,233 & 0,003 \\
Le ménage habite dans le quartier Khongolote & $0,757^{* * * *}$ & 0,301 & 0,012 \\
Le ménage habite dans le quartier Magoanine «A $\mathrm{A}$ & $0,882^{* * * * *}$ & 0,246 & 0,000 \\
Le ménage habite dans le quartier Magoanine «B $\mathrm{B}$ & $0,606^{* * * *}$ & 0,210 & 0,004 \\
Le ménage habite dans le quartier Magoanine « C » & $0,328^{* *}$ & 0,194 & 0,090 \\
Le ménage habite dans le quartier Albazine & 0,048 & 0,227 & 0,833 \\
Constante & $-0,312$ & 0,333 & 0,349 \\
\hline
\end{tabular}

Nombre d'observations : 871

Log pseudolikelihood : $-426,55941$

Wald $\mathrm{Chi}^{2}$ (20) : 216,48

Prob > $\mathrm{Chi}^{2}: 0,0000$

Pseudo $\mathrm{R}^{2}: 28,83 \%$

$\mathrm{R}^{2}$ de Mac Fadden : $28,8 \%$

AIC : 1,028

BIC : $-4901,077$

Cas correctement prédits : $75,89 \%$

$* * * * * * *, * *, *$ significatifs respectivement à $1 \%, 5 \%, 10 \%$ et $15 \%$. 
Tableau A8. Tableau de corrélation

\begin{tabular}{|c|c|c|c|c|c|c|c|c|}
\hline \multirow[b]{2}{*}{ Quartier } & \multicolumn{2}{|c|}{$\begin{array}{l}\text { Le ménage possède } \\
\text { un congélateur }\end{array}$} & \multicolumn{2}{|c|}{$\begin{array}{l}\text { Le ménage possède } \\
\text { un micro-onde }\end{array}$} & \multicolumn{2}{|c|}{$\begin{array}{c}\text { Le ménage possède } \\
\text { un ventilateur }\end{array}$} & \multicolumn{2}{|c|}{$\begin{array}{l}\text { Le ménage possède } \\
\text { un réfrigérateur }\end{array}$} \\
\hline & Oui & Non & Oui & Non & Oui & Non & Oui & Non \\
\hline Habel Jafar & 98 & 2 & 100 & 0 & 90 & 10 & 95 & 5 \\
\hline Matèque & 71 & 29 & 100 & 0 & 85 & 15 & 95 & 5 \\
\hline Guava & 73 & 27 & 93 & 7 & 80 & 20 & 80 & 20 \\
\hline Matola Gare & 60 & 40 & 83 & 17 & 50 & 50 & 50 & 50 \\
\hline Cumbeza & 93 & 7 & 100 & 0 & 95 & 5 & 97 & 3 \\
\hline Nkobe & 83 & 17 & 100 & 0 & 83 & 17 & 88 & 12 \\
\hline Kilomètre 15 & 93 & 7 & 97 & 3 & 92 & 8 & 90 & 10 \\
\hline $1^{\mathrm{er}}$ de Maio & 87 & 13 & 97 & 3 & 82 & 18 & 87 & 13 \\
\hline Ndlevela & 63 & 37 & 96 & 4 & 63 & 37 & 81 & 19 \\
\hline Khongolote & 62 & 38 & 92 & 8 & 73 & 27 & 74 & 26 \\
\hline Zimpeto & 51 & 49 & 90 & 10 & 50 & 50 & 63 & 37 \\
\hline Magoanine « $\mathrm{A} »$ & 50 & 50 & 95 & 5 & 56 & 44 & 70 & 30 \\
\hline Magoanine « $\mathrm{B}$ » & 49 & 51 & 84 & 16 & 49 & 51 & 50 & 50 \\
\hline Magoanine $« \mathrm{C} »$ & 70 & 30 & 96 & 4 & 70 & 30 & 74 & 26 \\
\hline Albazine & 63 & 37 & 94 & 6 & 73 & 27 & 82 & 18 \\
\hline
\end{tabular}


Tableau A9. Estimation avec l'indice de richesse remplacé par les variables d'équipement (variante 2)

\begin{tabular}{|c|c|c|c|}
\hline \multicolumn{4}{|c|}{ Variable dépendante : « Probabilité pour un ménage d'être branché à un réseau POP » } \\
\hline Variables explicatives & Coef. & Std. Err. & $\mathrm{P}>z$ \\
\hline Le chef de ménage est une femme (1/0) & 0,027 & 0,130 & 0,836 \\
\hline L'âge du chef de ménage & $-0,003$ & 0,005 & 0,620 \\
\hline Le chef de ménage est analphabète $(1 / 0)$ & $-0,541 * * * *$ & 0,202 & 0,007 \\
\hline Le ménage est locataire de son logement $(1 / 0)$ & $-0,615 * * *$ & 0,276 & 0,026 \\
\hline La taille du ménage & $0,058 * * *$ & 0,025 & 0,022 \\
\hline $\begin{array}{l}\text { Le ménage vit avec moins d'un dollar par jour } \\
\text { et par personne }\end{array}$ & $-0,477 * * * *$ & 0,114 & 0,000 \\
\hline Le ménage possède un congélateur & $0,656 * * * *$ & 0,126 & 0,000 \\
\hline Le ménage possède un micro-onde & $0,570 * *$ & 0,350 & 0,104 \\
\hline Le ménage possède un ventilateur & $0,350 * * * *$ & 0,131 & 0,008 \\
\hline Le ménage possède un réfrigérateur & $0,670 * * * *$ & 0,140 & 0,000 \\
\hline La part des branchés insatisfaits du service dans le quartier & $0,041 * * * *$ & 0,015 & 0,008 \\
\hline Le ménage habite dans le quartier Matèque & $1,358 * * * *$ & 0,387 & 0,000 \\
\hline Le ménage habite dans le quartier Guava & $-0,618 * * *$ & 0,301 & 0,040 \\
\hline Le ménage habite dans le quartier Matola Gare & $-0,558 * *$ & 0,351 & 0,112 \\
\hline Le ménage habite dans le quartier Cumbeza & $0,995 * * * *$ & 0,315 & 0,002 \\
\hline Le ménage habite dans le quartier Nkobe & $-0,123$ & 0,248 & 0,620 \\
\hline Le ménage habite dans le quartier $\mathrm{Km} 15$ & 0,016 & 0,289 & 0,955 \\
\hline Le ménage habite dans le quartier 1er de Maio & $0,843 * * * *$ & 0,242 & 0,000 \\
\hline Le ménage habite dans le quartier Ndlavela & $0,835^{* * * *}$ & 0,242 & 0,001 \\
\hline Le ménage habite dans le quartier Khongolote & $0,853 * * * *$ & 0,291 & 0,003 \\
\hline Le ménage habite dans le quartier Magoanine « $\mathrm{A}$ » & $0,987 * * * *$ & 0,240 & 0,000 \\
\hline Le ménage habite dans le quartier Magoanine « $\mathrm{B} »$ & $0,683 * * * *$ & 0,222 & 0,002 \\
\hline Le ménage habite dans le quartier Magoanine « $\mathrm{C} »$ & $0,294 * *$ & 0,192 & 0,125 \\
\hline Le ménage habite dans le quartier Albazine & 0,100 & 0,228 & 0,662 \\
\hline Constante & $-0,997 * * * *$ & 0,342 & 0,004 \\
\hline
\end{tabular}

Nombre d'observations : 852

Log pseudolikelihood : $-428,1244$

Wald $\mathrm{Chi}^{2}(24): 220,01$

Prob $>\mathrm{Chi}^{2}: 0,0000$

Pseudo $\mathrm{R}^{2}: 26,97 \%$

R2 de Mac Fadden : $27 \%$

AIC : 1,064

BIC : $-4724,005$

Cas correctement prédits : 73,59\%

$* * * *, * * *, * *, *$ significatifs respectivement à $1 \%, 5 \%, 10 \%$ et $15 \%$. 
Tableau A10. Estimation sans les quartiers (variante 3)

\begin{tabular}{l|c|c|c}
\hline \multicolumn{3}{c}{ Variable dépendante : « Probabilité pour un ménage d'être branché à un réseau POP » } \\
\hline \multicolumn{1}{c}{ Variables explicatives } & Coef. & Std. Err. & $\mathrm{P}>z$ \\
\hline Le chef de ménage est une femme (1/0) & 0,052 & 0,128 & 0,682 \\
L'âge du chef de ménage & $-0,002$ & 0,005 & 0,673 \\
Le chef de ménage est analphabète (1/0) & $-0,340^{* *}$ & 0,197 & 0,085 \\
Le ménage est locataire de son logement (1/0) & $-0,600^{* * *}$ & 0,255 & 0,019 \\
La taille du ménage & 0,018 & 0,024 & 0,452 \\
L'indice de richesse du ménage & $0,172^{* * * *}$ & 0,016 & 0,000 \\
Le ménage vit avec moins d'un dollar par jour et par & & & \\
personne & $-0,280^{* * * *}$ & 0,108 & 0,010 \\
La part des branchés insatisfaits du service dans le quartier & 0,006 & 0,007 & 0,337 \\
Constante & $0,316^{*}$ & 0,213 & 0,139 \\
\hline
\end{tabular}

Nombre d'observations : 871

Log pseudolikelihood : $-458,50499$

Wald $\mathrm{Chi}^{2}(8): 170,74$

Prob > Chi2 : 0,0000

Pseudo $\mathrm{R}^{2}: 23,50 \%$

$\mathrm{R}^{2}$ de Mac Fadden : 23,5 \%

AIC : 1,073

BIC : $-4918,421$

Cas correctement prédits : $73,82 \%$

$* * * *, * * *, * *, *$ significatifs respectivement à $1 \%, 5 \%, 10 \%$ et $15 \%$. 